\title{
Extraction of Oil from Oil Sands Using Thermo-Responsive Polymeric Surfactants
}

\author{
Bingqing Yang, Jean Duhamel*
}

Institute for Polymer Research, Waterloo Institute for Nanotechnology, Department of Chemistry, University of Waterloo, Waterloo, ON N2L 3G1, Canada

* To Whom correspondence should be addressed - jduhamel@uwaterloo.ca 


\section{ABSTRACT}

Several thermoresponsive block copolymers constituted of a poly(ethylene glycol) (PEG) and a poly(2-(2-methoxyethoxy) ethyl methacrylate) ( $\left.\mathrm{PMEO}_{2} \mathrm{MA}\right)$ block were prepared by Atom Transfer Radical Polymerization (ATRP) and their ability to extract oil from oil sands was evaluated. The chemical composition of the $\mathrm{PEG}_{113}-b-\mathrm{PMEO}_{2} \mathrm{MAx}$ block copolymers was determined by ${ }^{1} \mathrm{H}$ NMR and Gel Permeation Chromatography (GPC) with $X$-values ranging between 48 and 80 . Aqueous solutions of block copolymers showed a cloud point of $34 \pm$ $1{ }^{\circ} \mathrm{C}$ as determined by turbidimetry and dynamic light scattering (DLS) measurements. DLS experiments indicated that these polymers formed stable block copolymer micelles due to association of the $\mathrm{PMEO}_{2} \mathrm{MA}$ blocks at temperatures greater than $45{ }^{\circ} \mathrm{C}$ with a unimodal distribution of hydrodynamic diameters. Since characterization of the block copolymer solutions as a function of temperature indicated the formation of hydrophobic domains in water for $T>45{ }^{\circ} \mathrm{C}$, extractions of oil from oil sands with the block copolymers were conducted at $T=45$ and $50{ }^{\circ} \mathrm{C}$. At these temperatures, $15 \mathrm{~mL}$ of a $1 \mathrm{mg} / \mathrm{mL}$ PEG $113-b$ $\mathrm{PMEO}_{2} \mathrm{MA} 77$ aqueous solution extracted $100 \%$ of the oil trapped in $1 \mathrm{~g}$ of oil sand if $60 \mathrm{mg}$ of toluene was added to the mixture. When the extraction was conducted under the same experimental conditions without block copolymer, a poor oil recovery of less than $30 \%$ was achieved. Starting with a $1 \mathrm{mg} / \mathrm{mL}$ block copolymer concentration, the block copolymer aqueous solution could be recycled up to five successive extractions while maintaining satisfying oil recovery. Each extraction cycle led to a $22 \%$ mass loss of block copolymer, certainly due to association with the toluene, oil, and sand particles. Together these experiments demonstrate that thermoresponsive block copolymers can be powerful aids to enhance the oil recovery of oil sands. 
KEYWORDS: Thermoresponsive, lower critical solution temperature, block copolymer, micelles, oil extraction, oil sands.

\section{INTRODUCTION}

Oil sands, also known as tar or bitumen sands, are deposits of silica particles soaked in bitumen, a high molar mass viscous petroleum. ${ }^{1,2}$ Oil sands are found throughout the world. Canada and Venezuela have the world's two largest reserves of bitumen, with combined oil sands reserves estimated to be equal to the world's total reserves of conventional crude oil. ${ }^{1,3}$ The largest deposit, and the only one of present commercial importance is in the Athabasca region located in the northeastern part of Alberta, Canada. The extraction of bitumen from oil sands is of high economic interest but presents some difficult challenges. Since bitumen is a heavy oil consisting of large hydrocarbon molecules that are usually in the solid state at room temperature, ${ }^{4}$ their extraction is difficult. In addition, the Athabasca region possesses 250 billion barrels worth of bitumen located in beds of sand and clay where the oil and sand usually stick to each other ${ }^{2,4}$ further complicating the oil extraction process.

For more than one hundred years, numerous scientists, engineers, and entrepreneurs have investigated how the bitumen can be recovered from the oil sands economically and efficiently. Several bitumen extraction processes have been developed over the years and these processes can generally be divided into two main families. The first family is referred to as open pit mining technology, ${ }^{1}$ where the oil sands are mined and transported to a processing plant where the bitumen is extracted. The second family aims to separate the bitumen from the sand directly in the geological formation without moving the sand and it is referred to as insitu technology. ${ }^{1}$ 
The methods applied for in-situ technology always require a high temperature since this technology uses heat to melt the bitumen trapped in the oil sands, allowing it to flow until it can be efficiently collected. Examples of in-situ processes include fire flooding, ${ }^{5}$ a combination of forward combustion and water flood referred to as the COFCAW process, ${ }^{6}$ or the emulsion-steam driven process. ${ }^{7}$ Like the in-situ technology, open mining also requires energy for bitumen extraction. The first reported commercial process for the extraction of bitumen from Athabasca oil sands was the Clark Hot Water Extraction (CHWE) process developed by Karl Clark in the 1920s ${ }^{1,2}$ Interestingly, most companies involved in Alberta bitumen extraction today still use variations of the CHWE with operation temperatures ranging between 50 and $80{ }^{\circ} \mathrm{C}$. Beside the $\mathrm{CHWE}$ process, direct coking of the oil sands ${ }^{1}$ is the most straightforward bitumen extraction method, whereby the oil sand is heated up by contact with a bed of clean sand in a coker or still maintained at temperatures that range from 480 to $760{ }^{\circ} \mathrm{C}$. In the 1990 s, a cold water process ${ }^{8,9}$ was introduced by Sury to lower the temperature of bitumen extraction. This method used water with a conditioning agent that is mixed with the oil sands at temperatures ranging from 5 to $25^{\circ} \mathrm{C}$.

Water-based extraction of bitumen from Athabasca oil sands causes environmental issues, regardless of whether it is based upon in situ or open pit mining technologies. ${ }^{2,10}$ First, the heat necessary for bitumen extraction causes greenhouse gas emission, which is a known contributor to global warming. ${ }^{2,10}$ Second, tailing ponds are oil-in-water emulsions that are generated by the bitumen extraction. They have had an adverse impact on the local environment. ${ }^{2,10}$ Since water-based bitumen extraction consists of a sequence of mining (for open mine techniques), extraction, froth treatment, and water management in the tailing 
ponds, ${ }^{11}$ there is a demand for new extraction processes or froth treatments that would mitigate or even eliminate those steps along the extraction path that are detrimental to the environment.

Considering the bitumen froth treatment, it must be pointed out that a typical bitumen froth is composed of $60 \mathrm{wt} \%$ bitumen, $30 \mathrm{wt} \%$ water, and $10 \mathrm{wt} \%$ mineral solids. ${ }^{11-13}$ In the last twenty years, notable progress has been made in the handling of bitumen froth to enhance overall bitumen recovery and reduce the number of tailing ponds. Paraffinic (PFT) and naphthenic (NFT) froth treatments are two of the methods that are applied in industrial operations. Naphthenic and paraffinic solvents are employed to increase the organic content of the bitumen froth and lower its viscosity so that the inorganic impurities (water and mineral particles) can be separated from the solution of bitumen and organic solvent. As compared to PFT, the NFT process consumes much more energy but recovers bitumen with a higher yield. Due to the higher recovery, NFT has been applied to most projects of the Athabasca oil sands industry. Although both methods enhance bitumen recovery and generate fewer tailing ponds, both PFT and NFT use large quantities of organic solvent, such as paraffin and naphtha which are environmentally unfriendly and more difficult to deal with as compared to water. ${ }^{11-13}$

The design of new procedures aiming at improving oil extraction efficiency while reducing their detrimental impact on the environment could either result in an entire rethink of the existing technology, or draw from the main features of current extraction technology such as the use of surfactant, organic thinner, water, and heat to combine them in a manner that would yield a more efficient extraction process. It was this latter line of thoughts that was followed in the present study that investigates how an aqueous solution of a thermoresponsive polymeric surfactant would, in the presence of a small amount of organic thinner, 
affect the efficiency of oil extraction from oil sands. Considering the large body of work found in the scientific literature on block copolymers where the heat-induced insolubility of a selected block results in the formation of block copolymer micelles, ${ }^{14,15}$ we selected a poly(ethylene-glycol)-b-poly(2-(2-methoxyethoxy)ethyl methacrylate] diblock copolymer (PEG- $b$-PMEO $2 \mathrm{MA}$ ) as a thermo-responsive polymeric surfactant. ${ }^{16}$ While PEG is watersoluble between 0 and $98{ }^{\circ} \mathrm{C}, \mathrm{PMEO}_{2} \mathrm{MA}$ has an LCST of $26{ }^{\circ} \mathrm{C} .{ }^{17}$ Consequently, PEG-b$\mathrm{PMEO}_{2} \mathrm{MA}$ was expected to be completely water-soluble or form block copolymer micelles with a $\mathrm{PMEO}_{2} \mathrm{MA}$ core below or above the LCST of the $\mathrm{PMEO}_{2} \mathrm{MA}$ block, respectively.

Taking advantage of the thermo-responsiveness of PEG- $b-\mathrm{PMEO}_{2} \mathrm{MA}$ in aqueous solution, the extraction scheme depicted in Figure 1 was implemented. To $15 \mathrm{~mL}$ of a $1 \mathrm{mg} / \mathrm{mL}$ aqueous solution of PEG-b-PMEO $2 \mathrm{MA}$ at room temperature $\left(\mathrm{RT}=23{ }^{\circ} \mathrm{C}\right)$ below the cloud point $(T \mathrm{C})$ of the polymer solution was introduced $1 \mathrm{~g}$ of oil sands supplied to us by Imperial Oil. The oil sand paste sank to the bottom of the solution and a small amount of toluene (typically $60 \mathrm{mg}$ ) was deposited at the surface of the aqueous solution. The mixture was then placed in a shaker and left shaking overnight at $45{ }^{\circ} \mathrm{C}$ which was above the cloud point of the block copolymer solution. After this treatment, the mixture was cooled to RT and the following observations were made on the mixture. The pristine sand was found at the bottom of the vial, the aqueous solution was turbid due to unsettled sand particles, and the oil laced with toluene had gathered at the surface of the water phase where it could be skimmed off. Within experimental error, $100 \%$ of the oil found in the oil sand was recovered and the aqueous solution retained $80 \%$ of the block copolymer so that it could be re-used for several additional rounds of extraction. These claims are illustrated in Figure 2 that shows the mixture before and after the extraction. 


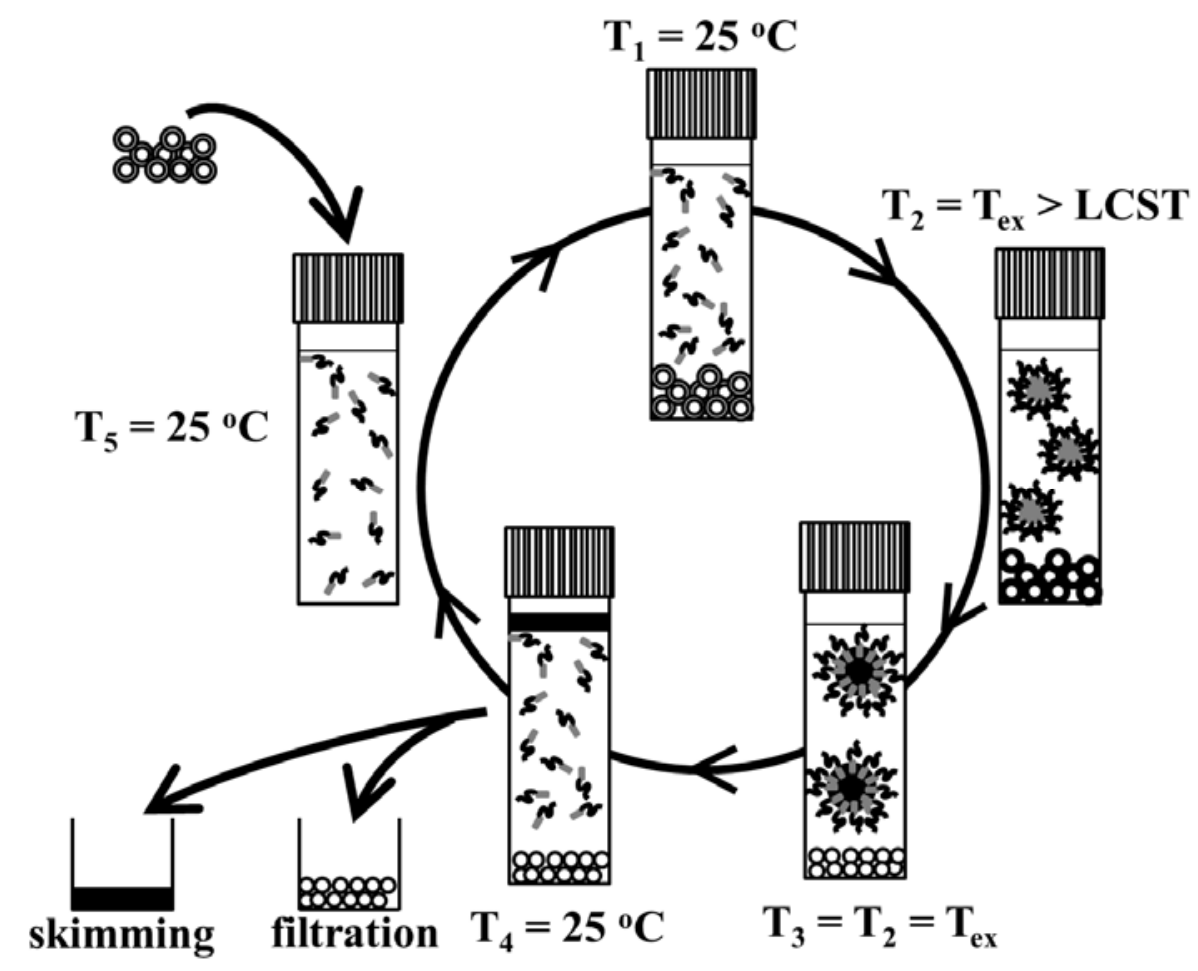

Figure 1. Proposed process for oil extraction from oil sands by using the thermo-responsive block copolymer PEG- $b-\mathrm{PMEO}_{2} \mathrm{MA}$.

While the use of a block copolymer such as PEG- $b-\mathrm{PMEO}_{2} \mathrm{MA}$ is highly unlikely to be adopted by the oil extraction industry due to the relative chemical complexity of this thermo-responsive polymeric surfactant, the results described in this study clearly illustrate the potential that the use of thermo-responsive polymeric surfactants might hold to improve the oil extraction efficiency for oil sands. Considering the strong industrial interest residing in enhancing the notoriously challenging oil extraction from oil sands, this study is expected to open new research venues toward achieving this goal with thermo-responsive polymeric surfactants. The preparation and characterization of the thermo-responsive polymeric 
surfactant, its solution properties, and the experiments that were conducted to identify its efficiency at extracting oil from oil sands are described in detail hereafter.

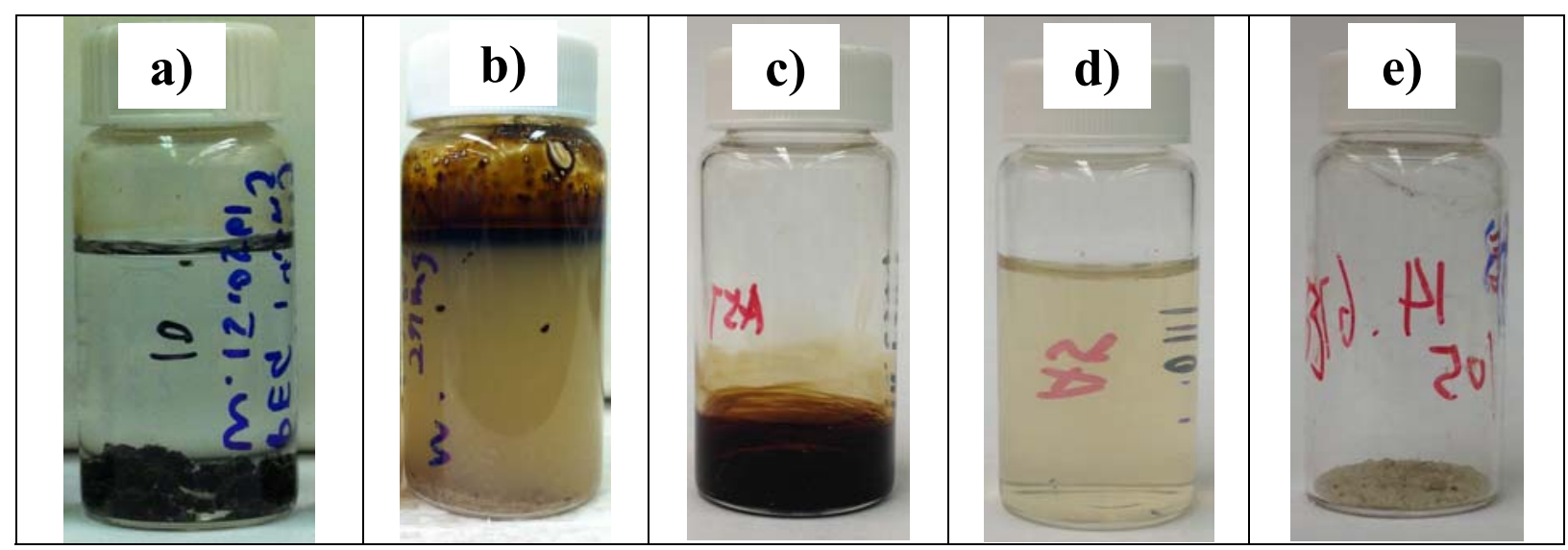

Figure 2. a) Before extraction: Mixture of oil sand particles at the bottom, $15 \mathrm{~mL}$ of $1 \mathrm{mg} / \mathrm{mL}$ $\mathrm{PEG}_{113}-b-\mathrm{PMEO}_{2} \mathrm{MA}_{77}$ aqueous solution, and $60 \mathrm{mg}$ of toluene on top of the solution. After $24 \mathrm{hrs}$ extraction at $\mathrm{T}=45^{\circ} \mathrm{C}$ : b) Mixture with the extracted oil at the top of the aqueous solution; c) Oil recovered from the extraction; d) $\mathrm{PEG}_{113}-b-\mathrm{PMEO}_{2} \mathrm{MA} 77$ aqueous solution; e) Pristine sand particles.

\section{EXPERIMENTALS}

Materials. 2-Bromoisobutyryl bromide $(\geq 98 \%), \quad N, N, N, N ", N$ "-pentamethyldiethylenetriamine (PMDETA, $\geq 99 \%$ ), 2-(2-methoxyethoxy)ethyl methacrylate $\left(\mathrm{MEO}_{2} \mathrm{MA}, 95 \%\right)$, magnesium sulfate $\left(\mathrm{MgSO}_{4}, \geq 99.5 \%\right), 2,2$ '-bipyridine $(\geq 98 \%)$, toluene $(\geq 99.9 \%)$, tetrahydrofuran (THF, $\geq 99.5 \%$ ), $n$-hexane ( $\geq 98.5 \%$ ), methanol ( $\geq 99.9 \%$ ), diethyl ether ( $\geq 99.0 \%$ ), ethanol (HPLC Grade), dichloromethane (DCM, $\geq 99.8 \%$ ), PEG terminated with a hydroxyl group at one end and a methyl group at the other end (Me-PEG-OH, $M_{\mathrm{n}}=$ 5000 g.mol ${ }^{-1}$ ), and $\mathrm{CuBr}(99.999 \%)$ were purchased from Aldrich and used as received unless 
otherwise noted. Me-PEG-OH was purified by dissolution in DCM followed by precipitation with cold diethyl ether. The precipitation was repeated twice. Gel permeation chromatography (GPC) analysis in THF with polystyrene standards showed that the Me-PEG-OH sample had a narrow molecular weight distribution $(\mathrm{PDI}=1.1$ in Table 1$)$ and after its complete reaction with 2-bromoisobutyryl bromide, ${ }^{1} \mathrm{H}$ NMR analysis yielded an $M_{\mathrm{n}}$ value of 5,130 g/mol. THF and ethanol were distilled prior to use. $\mathrm{CuBr}$ was washed with deionized water, acetic acid (Fisher, ACS reagent, glacial), ethanol, and diethyl ether in that sequence and then dried in vacuum and stored under nitrogen before use. Milli-Q Millipore filtered water (18 M $\Omega . \mathrm{cm})$ was used in all experiments. Praxair Ultra Pure 5.0 nitrogen was used in all syntheses. The synthesis of the 2-bromoisobutyrate PEG macroinitiator containing 113 ethylene glycol units and the $\mathrm{PEG}_{113}-b-\mathrm{PMEO}_{2} \mathrm{MAx}$ samples with $X=48,64,77$, and 80 were carried out according to reported procedures ${ }^{17,18}$ and are described in detail in the next section. Imperial Oil supplied a sample of oil sands.

Synthesis of 2-Bromopropionate PEG Macroinitiator. A poly(ethylene glycol) PEG macroinitiator was first prepared according to a published procedure (Scheme 1). ${ }^{18}$ The hydroxyl end group of Me-PEG-OH was reacted with 2-bromoisobutyryl bromide to give the 2-bromoisobutyrate PEG macroinitiator.

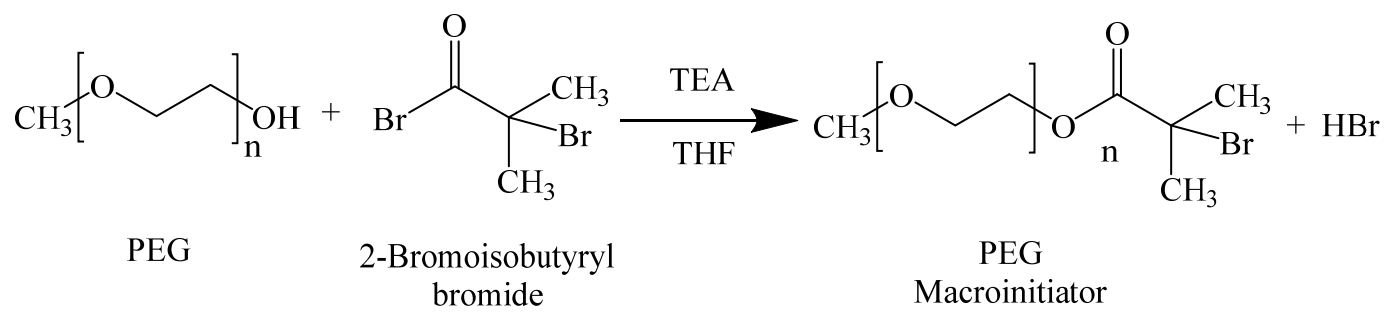

Scheme 1: Synthesis of PEG macroinitiator. ${ }^{16,18}$ 
Me-PEG-OH (5.5 g, $1.1 \mathrm{mmol})$ was dissolved in freshly distilled tetrahydrofuran (THF, 50 $\mathrm{mL}$ ) in a three-neck round bottom flask that had been dried beforehand by flaming under vacuum followed by purging with nitrogen. Triethylamine (TEA, $0.46 \mathrm{~mL}, 3.3 \mathrm{mmol}$ ) was then added under nitrogen. The flask was lowered in an ice-water bath at $0{ }^{\circ} \mathrm{C}$ and 2 bromoisobutyryl bromide $(0.82 \mathrm{~mL}, 6.6 \mathrm{mmol})$ was injected by using a glass pipette into the reaction flask. All the processes were conducted under a positive nitrogen pressure to prevent the introduction of moisture from the air. After the addition of 2-bromoisobutyryl bromide, the reaction was stirred at room temperature (RT) for $24 \mathrm{hrs}$. During the reaction, a precipitate of TEA hydrobromide formed. The precipitate was removed by centrifugation. Magnesium sulfate $(\sim 0.2 \mathrm{~g})$ was added to remove any traces of water that might be absorbed by the mixture during the centrifugation process. A clear solution was collected. Finally, the macroinitiator was purified with 4 cycles of precipitation into $n$-hexane at $-72{ }^{\circ} \mathrm{C}$ (by keeping the vessel on dry ice), filtration, and drying under vacuum.

Synthesis of PEG-bPMEO ${ }_{2} M A$ by ATRP. With the PEG macroinitiator, a conventional ATRP procedure was applied to synthesize the PEG-b-PMEO 2 MA copolymer according to Scheme $2 .^{16,19}$

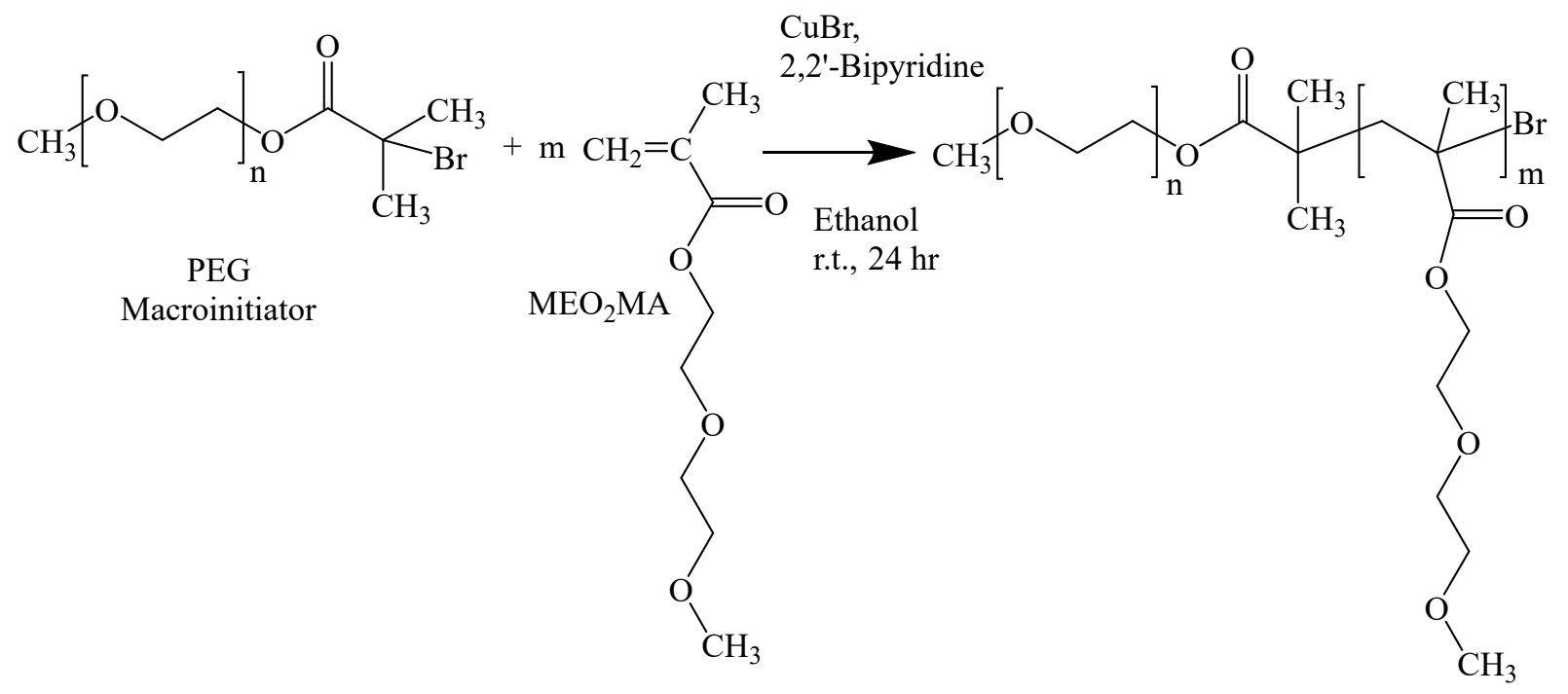


Scheme 2: Synthesis of PEG-b-PMEO 2 MA by ATRP. ${ }^{16,19}$

The 2-bromoisobutyrate PEG macroinitiator (0.990 g, $0.192 \mathrm{mmol})$, 2-(methoxyethoxy)ethyl methacrylate ( $\left.\mathrm{MEO}_{2} \mathrm{MA}, 2.7 \mathrm{~mL}, 15 \mathrm{mmol}\right)$, and 2,2'-bipyridine $(91.2 \mathrm{mg}, 0.584 \mathrm{mmol})$ were dissolved in ethanol $(3.3 \mathrm{~mL})$ and the solution was placed in a Schlenk tube. The mixture was degassed by three freezing-evacuation-thawing cycles. In the last cycle, the Schlenk tube was filled with $\mathrm{N}_{2}$ and the mixture was kept frozen. The catalyst $\mathrm{CuBr}(42.0 \mathrm{mg}, 0.293 \mathrm{mmol})$ was added as a fine powder through a Pasteur pipette to the surface of the frozen solid against a positive pressure of nitrogen. After addition of the catalyst, the mixture was degassed with one more freezing-evacuation-thawing cycle. Finally, the tube was filled with $\mathrm{N}_{2}$, tightly sealed, and stirred at RT for $24 \mathrm{hrs}$.

After $24 \mathrm{hrs}$, the reaction was terminated by purging the vessel with air. The ethanol was left to evaporate. The resulting oily mixture was dissolved in methanol. ${ }^{16,19}$ Then the brownish mixture was passed through a short $(3-5 \mathrm{~cm})$ silica gel column (neutral, $40-60 \mu \mathrm{m}$ ) (eluent, methanol) to remove the copper complex. ${ }^{18}$ Finally, the product was dialyzed in a regenerated cellulose membrane tubing (molecular weight cut-off, 8,000; Spectrum, Rancho Dominguez, CA) against methanol for several days to remove small molecules. The methanol was removed with a rotary evaporator, and the polymer sample was dried under vacuum at room temperature.

Gel Permeation Chromatography (GPC). The molecular weight distribution (MWD) of the polymers was determined at ambient temperature with a Viscotek GPCmax VE2001 from Malvern instruments Ltd. (UK) using THF as the eluent. The GPC instrument was equipped with a right-angle light scattering (RALS), low-angle light scattering (LALS), ultraviolet 
absorbance (UV), and differential refractive index (DRI) detectors. Because the PEG113-b$\mathrm{PMEO}_{2} \mathrm{MAx}$ samples did not scatter enough light to determine their absolute molecular weight based on GPC analysis, the apparent molecular weight of these samples was reported using a calibration curve based on polystyrene (PS) standards.

${ }^{1} H$ NMR Spectroscopy. It was used to confirm the chemical composition of the PEG- $b$ $\mathrm{PMEO}_{2} \mathrm{MA}$ block copolymers by determining their absolute number average molecular weight $\left(M_{\mathrm{n}}\right)$.

Turbidity Measurements. The transmittance of a $5 \mathrm{mg} / \mathrm{mL}$ polymer aqueous solution was monitored at $400 \mathrm{~nm}$ by using a UV-Vis spectrophotometer (Model CARY-100, Welltech Enterprises, INC., Maryland, US). The temperature was increased at a rate of $0.5^{\circ} \mathrm{C} / \mathrm{min}$. The turbidimetry profiles showed a constant transmittance equal to $100 \%$ at temperatures lower than the cloud point before undergoing an abrupt drop at $T_{\mathrm{CP}} . T_{\mathrm{CP}}$ was taken as the temperature corresponding to the intersection between the horizontal line at $100 \%$ before the breakpoint and the straight line drawn to represent the large decrease in transmittance after the breakpoint. $^{20}$

Dynamic Light Scattering (DLS) Measurements. After dissolution of the polymer in water at a concentration of $5 \mathrm{mg} / \mathrm{mL}$, the solution was centrifuged at 13,300 rpm for $10 \mathrm{~min}$ to remove dust particles. Then, the hydrodynamic diameter $\left(d_{\mathrm{h}}\right)$ of the polymer species present in solution was measured as a function of temperature with a Brookhaven 90 Plus particle sizer (Brookhaven Instruments, Inc., Holtzville, NY), which measures the scattered light at a $90^{\circ}$ angle, or a Zetasizer Nano-ZS (Malvern Instruments Ltd., Worcestershire, UK), which measures the scattered light at a $173^{\circ}$ angle. 
Viscosity Measurements. Solutions were prepared at polymer concentrations ranging between 5 and $25 \mathrm{mg} / \mathrm{mL}$. The viscosity of the solutions was determined at $50{ }^{\circ} \mathrm{C}$ with an Ubbelohde viscometer (Model D504, Cannon Instrument Company, State College, PA).

Soxhlet Extraction. To quantify the total mass of oil recovered from one gram of oil sands, Soxhlet extraction was applied following a protocol established by Jacobs and Filby. ${ }^{21}$ Approximately $5 \mathrm{~g}$ of oil sands wrapped in filter paper was placed inside the main chamber of the Soxhlet apparatus. Then the apparatus was used to extract the bitumen from the oil sand sample using refluxing toluene $\left(T_{b}=110^{\circ} \mathrm{C}\right)$ or THF $\left(T_{b}=66^{\circ} \mathrm{C}\right)$ as the solvent. The round bottom flask of the apparatus was immersed in an oil bath which was heated and stirred with a magnetic stirrer. For both the toluene and THF extractions, the set up was left to reflux for 24 hrs. After the extraction was complete, the clean sand was dried in a vacuum oven at room temperature for $24 \mathrm{hrs}$, and the bitumen from the oil sand sample that had dissolved in toluene or THF was dried under a stream of nitrogen before placing it in a vacuum oven at room temperature for 24 hrs to remove any residual solvent. Regardless of the solvent used in the Soxhlet extraction, the oil sand samples were found to be constituted of $11 \pm 1 \mathrm{wt} \%$ of oil and $89 \pm 1 \mathrm{wt} \%$ of sand.

Extraction Protocol. At the bottom of a $20 \mathrm{~mL}$ scintillation vial, $1 \mathrm{~g}$ of oil sand was deposited before adding $15 \mathrm{~mL}$ of aqueous solutions of different polymers. Toluene $(25 \mathrm{mg}-150 \mathrm{mg})$ was placed on top of the aqueous solution (cf. Figure 2). The vials were placed in an incubator shaker (Innova 4000, New Brunswick Scientific Co., Inc., Nijmegen, Netherlands) where they were stirred at $250 \mathrm{rpm}$ at $45{ }^{\circ} \mathrm{C}$ or $50^{\circ} \mathrm{C}$. After $24 \mathrm{hrs}$ the shaker was stopped, the vial was taken out, and a picture was taken. 
Separation of Oil and Sand after Extraction. After the vials were taken out of the shaker, the oil present at the top of the aqueous solution and on the vial wall was recovered by rinsing the wall with a few drops of toluene and collecting the oil-loaded toluene with a Pasteur pipette. The toluene was evaporated under a gentle flow of nitrogen. Then the aqueous solution was removed and the oil that remained stuck to the sand at the bottom of the vial was collected by rinsing the oily sand with THF. The oil recovered in the top layer, the oil recovered in the bottom sand layer, and the sand free from oil were placed in a vacuum oven at room temperature overnight to remove any traces of water, THF, or toluene. The mass of sand and oil recovered after extraction were added and the total mass was compared to that of the mass of oil sands that was weighed originally. In $5 \%$ of all experiments, the two masses were found to differ by more than $\pm 10 \%$. In these few instances, the results were simply discarded. In all other cases, the mass of oil recovered on top of the aqueous phase was reported as the mass of oil extracted from the oil sands. Dividing the mass of oil per gram of oil sands recovered in an extraction by $0.11 \mathrm{~g} / \mathrm{g}$, the known amount of oil trapped in $1 \mathrm{~g}$ of oil sand sample determined by Soxhlet extraction, yielded the extraction efficiency $\left(E_{\mathrm{ex}}\right)$.

Recovery of $P E G_{113}-b-P M E O_{2} M A x$ after Extraction. After an oil extraction cycle was completed, the toluene layer laced with oil was removed and the aqueous layer was collected. To remove small sand particles that might have been introduced in the aqueous solution during the oil extraction process, the aqueous solution was centrifuged at room temperature at $10,000 \mathrm{rpm}$ for $15 \mathrm{~min}$. The supernatant was collected and freeze-dried for two days to remove the water. After removal of water, a white cotton-like solid was recovered which was dissolved in a known amount of THF. The solution was injected into the GPC and the DRI signal of the $\mathrm{PEG}_{113}-b-\mathrm{PMEO}_{2} \mathrm{MAx}$ solution in THF was measured to determine the 
concentration of the $\mathrm{PEG}_{113}-b-\mathrm{PMEO}_{2} \mathrm{MAx}$ copolymer in the THF solution using a calibration curve relating the DRI signal intensity to the $\mathrm{PEG}_{113}-b-\mathrm{PMEO}_{2} \mathrm{MAx}$ concentration in THF. The calibration curve was established by injecting solutions of known block copolymer concentration into the GPC instrument and plotting the maximum DRI intensity in the GPC trace as a function of polymer concentration. This plot yielded a straight line (see Figure S3 in SI) which was used as a calibration curve to determine the unknown concentration of the PEG- $b-\mathrm{PMEO}_{2} \mathrm{MA}$ solutions in THF that were injected into the GPC.

\section{RESULTS AND DISCUSSION}

Synthesis of a Thermo-Responsive Polymeric Surfactant. The PEG macroinitiator was synthesized by reacting the hydroxyl end group of Me-PEG-OH with 2-bromoisobutyryl bromide. ${ }^{18}$ The ${ }^{1} \mathrm{H}$ NMR spectrum and GPC traces confirmed the successful preparation of the PEG macroinitiator. Figure 3 shows the ${ }^{1} \mathrm{H}$ NMR spectrum of the purified PEG macroinitiator. Each proton of the macroinitiator could be assigned in the ${ }^{1} \mathrm{H}$ NMR spectrum. The absolute molecular weight of the PEG macroinitiator could be calculated based on the integrated intensities of peaks B and C. The number-average degree of polymerization, $\mathrm{DP}_{\mathrm{n}}$, of PEG was found to equal 113 resulting in an absolute molecular weight of 5,130 g/mol. The enlarged spectrum around $4.5 \mathrm{ppm}$ in the inset of Figure 3 showed that the broad peak at $4.5 \mathrm{ppm}$ representing the hydroxyl end group of PEG in $\mathrm{d}_{6}$-DMSO had disappeared, ${ }^{22}$ further confirming the successful synthesis of the 2-bromoisobutyrate PEG macroinitiator. In addition, the GPC trace of the purified product showed a single peak that appeared at the same elution volume as the single peak of the unmodified PEG, indicating that the size of the purified product was the same as that of the unmodified PEG. Therefore, it could be 
concluded that the synthesis of the 2-bromoisobutyrate PEG macroinitiator was successful, as confirmed by ${ }^{1} \mathrm{H}$ NMR and GPC analysis.

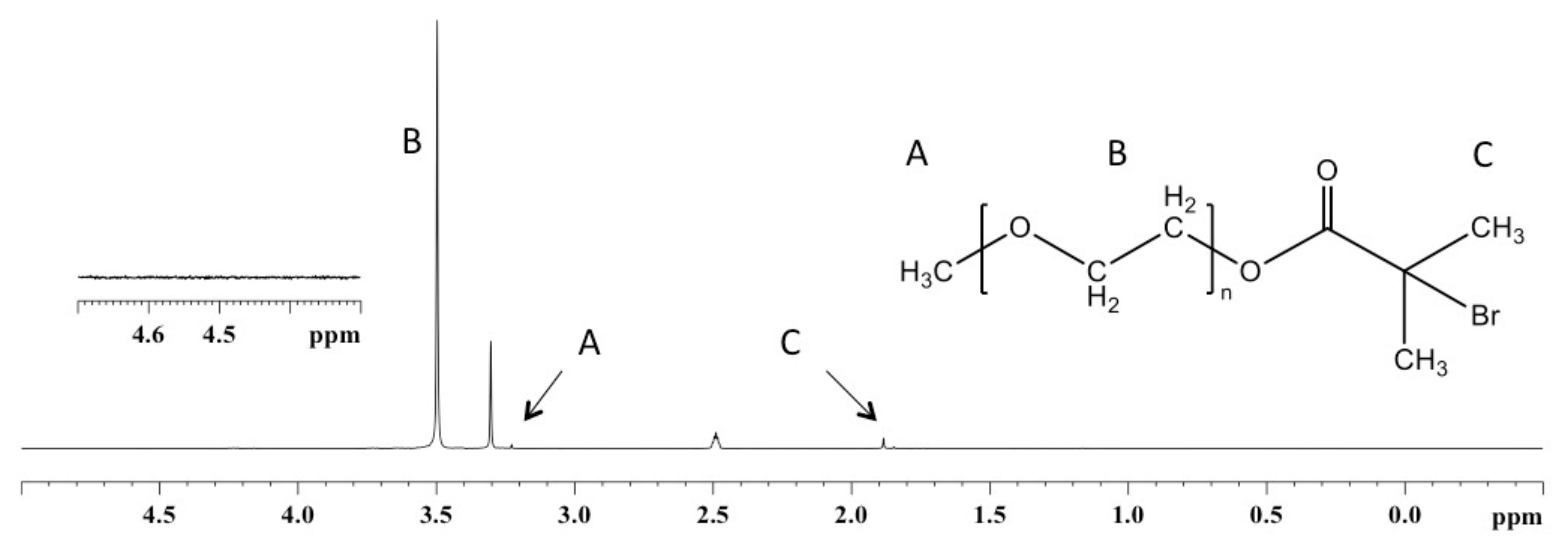

Figure 3. ${ }^{1} \mathrm{H}$ NMR spectrum of the purified 2-bromoisobutyrate PEG macroinitiator together with peak assignment. The small peak at $2.5 \mathrm{ppm}$ is for DMSO. [Poly] $=20 \mathrm{mg} / \mathrm{mL}$.

The synthesis of $\mathrm{PEG}_{113}-b-\mathrm{PMEO}_{2} \mathrm{MAx}$ was conducted by conventional ATRP using copper (I) bromide as catalyst and 2,2'-bipyridine as ligand. The polymerization was carried out in ethanol at room temperature for $24 \mathrm{hrs}$ as described in Scheme 2. As for the 2-bromoisobutyrate PEG macroinitiator, the synthesis of the block copolymer PEG113-b$\mathrm{PMEO}_{2} \mathrm{MAx}$ was confirmed by GPC measurements and ${ }^{1} \mathrm{H}$ NMR spectroscopy. The ${ }^{1} \mathrm{H}$ NMR spectrum for the purified product is shown in Figure 4.

Figure 4 indicates that the ratio of the peak areas related to protons $e$ and $B+b+c+d$ can be expressed as a function of $n$ and $m$ which are the number-average degrees of polymerization of the PEG and $\mathrm{PMEO}_{2} \mathrm{MA}$ blocks, respectively. The relationship between the NMR signal $(I)$ of the different protons and the degrees of polymerization $n$ and $m$ is shown in Equation 1. 


$$
r=\frac{I_{e}}{I_{B}+I_{b}+I_{c}+I_{d}}=\frac{2 m}{4 n+6 m}
$$

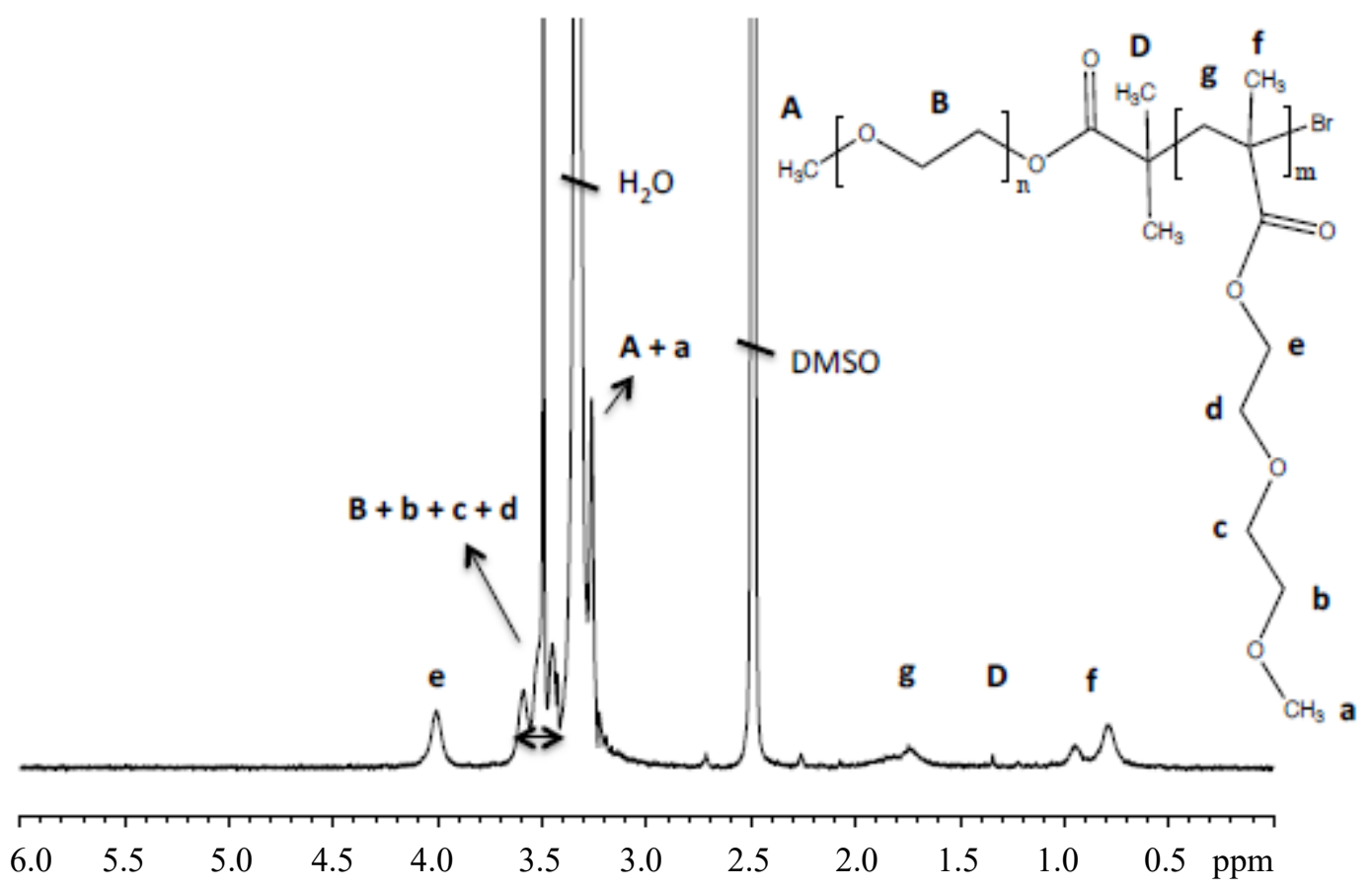

Figure 4. Chemical structure and ${ }^{1} \mathrm{H}$ NMR spectrum of $\mathrm{PEG}_{113}-b-\mathrm{PMEO}_{2} \mathrm{MA} 77$.

Isolating for the degrees of polymerization $n$ and $m$ yielded the relationship shown in Equation 2.

$$
m=\frac{2 r}{1-3 r} \times n
$$

Since $n$ was found to equal 113 from the analysis of the ${ }^{1} \mathrm{H}$ NMR spectrum obtained for the macroinitiator (Figure 3), Equation 2 yields the degree of polymerization of the $\mathrm{PMEO}_{2} \mathrm{MA}$ block of the thermo-responsive polymeric surfactants that were prepared for this study and they are given in Table 1. 
The GPC traces of the different polymeric constructs obtained during the synthesis of $\mathrm{PEG}_{113-b}-\mathrm{PMEO}_{2} \mathrm{MA} 77$ are shown in Figure 5. The narrow peak eluting at $22 \mathrm{~mL}$ in Figure 5A represents the $\mathrm{PEG}_{113}-b-\mathrm{PMEO}_{2} \mathrm{MA}_{77}$ copolymer while the spurious peaks appearing at elution volumes greater than $27 \mathrm{~mL}$ are associated with small molecules that elute with the solvent. The apparent molecular weight based on polystyrene standards was determined to equal $20,000 \pm 200 \mathrm{~g} / \mathrm{mol}$ with a PDI of $1.54 \pm 0.01$. Demonstration of chain extension of the macroinitiator is shown in Figure 5B which enlarges the area of the GPC traces around the elution volumes representative of the macroinitiator and the block copolymer. In Figure 5B, the peak eluting at $23.5 \mathrm{~mL}$ representing the PEG macroinitiator (trace a) was absent in the GPC trace of the purified product (trace c), which further confirmed the successful extension of the PEG macroinitiator into the corresponding block copolymer.

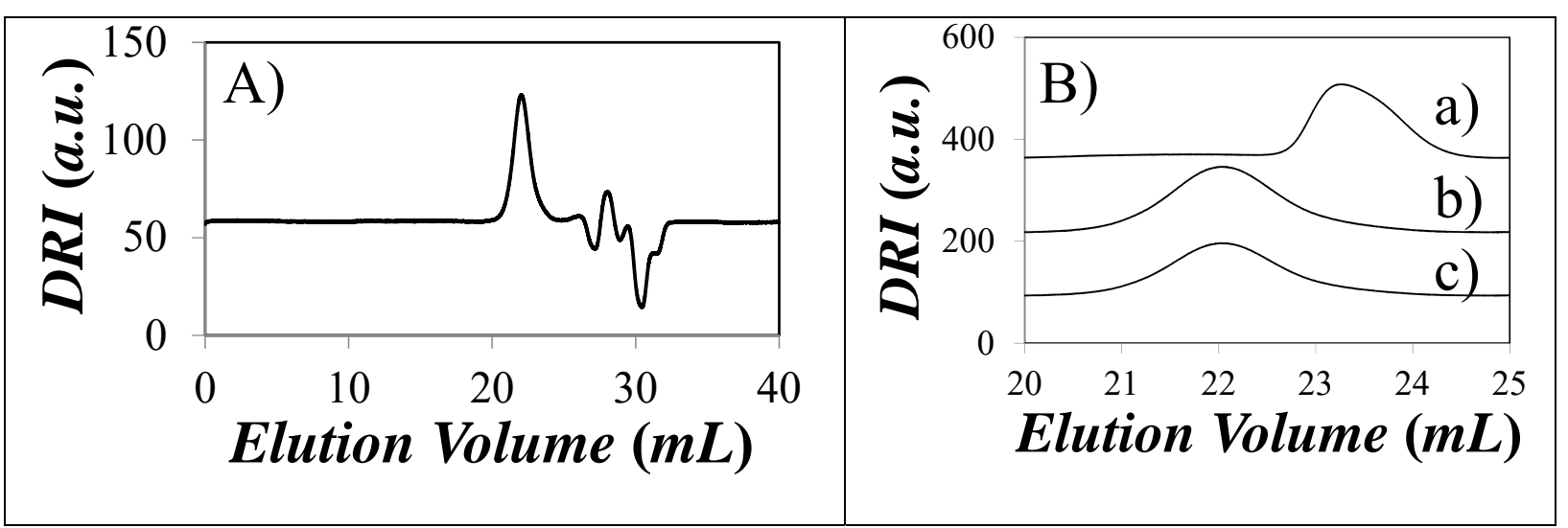

Figure 5. A) GPC trace for purified $\mathrm{PEG}_{113}-b-\mathrm{PMEO}_{2} \mathrm{MA}_{77}$. B) Zoomed-in GPC traces for (a) the macroinitiator, (b) the unpurified $\mathrm{PEG}_{13}-b-\mathrm{PMEO}_{2} \mathrm{MA}_{77}$ sample, and (c) the purified $\mathrm{PEG}_{113}-b-\mathrm{PMEO}_{2} \mathrm{MA}_{77}$ sample.

The characteristics of all the polymers synthesized by ATRP for this study are listed in Table 1. As the polymers were consumed during the course of the study, new polymers were 
synthesized as the need arose. Polymers 4, 5, and 6 were used to study micelle formation by the thermo-responsive polymeric surfactants. Polymers 5 and 6 were employed for the oil extraction experiments. 
Table 1. Summary of the polymers used in this study, their cloud points, and hydrodynamic diameter $\left(d_{\mathrm{h}}\right)$ at $T>T_{\mathrm{CP}}$.

\begin{tabular}{|c|c|c|c|c|c|c|c|}
\hline$\#$ & Polymer & $\begin{array}{c}\left.\mathrm{M}_{\mathrm{n}, \mathrm{NMR}}{ }^{\mathrm{a}}\right) \\
(\mathrm{g} / \mathrm{mol})\end{array}$ & $\begin{array}{c}\left.\mathrm{PDI}^{\mathrm{b}}\right) \\
(\text { by GPC) }\end{array}$ & $\begin{array}{l}\text { Weight \% } \\
\text { of PEG }\end{array}$ & $\begin{array}{c}T_{\mathrm{CP}}{ }^{\mathrm{c})} \\
\text { turbidimetry } \\
\left({ }^{\circ} \mathrm{C}\right)\end{array}$ & $\begin{array}{l}\left.T_{\mathrm{CP}}{ }^{\mathrm{c}}\right) \\
\mathrm{DLS} \\
\left({ }^{\circ} \mathrm{C}\right)\end{array}$ & $\begin{array}{l}d_{\mathrm{h}}{ }^{\mathrm{c})} \\
(\mathrm{nm})\end{array}$ \\
\hline 1 & $\mathrm{PMEO}_{2} \mathrm{MA}_{138}$ & 26000 & 1.8 & 0 & 26 & - & - \\
\hline 2 & $\mathrm{PEG}_{113}$ (Aldrich) & 5000 & 1.1 & 100 & - & - & - \\
\hline 3 & $\mathrm{PEG}_{113}-b-\mathrm{PMEO}_{2} \mathrm{MA}_{48}$ & 14000 & 1.5 & 36 & - & - & - \\
\hline 4 & $\mathrm{PEG}_{113}-b-\mathrm{PMEO}_{2} \mathrm{MA}_{64}$ & 17000 & 1.1 & 29 & 33 & $33 \pm 1$ & $26.3 \pm 0.3$ \\
\hline 5 & $\mathrm{PEG}_{113}-b-\mathrm{PMEO}_{2} \mathrm{MA}_{77}$ & 19000 & 1.5 & 26 & 35 & $30 \pm 5$ & $36.6 \pm 0.3$ \\
\hline 6 & $\mathrm{PEG}_{113}-b-\mathrm{PMEO}_{2} \mathrm{MA} 80$ & 20000 & 1.2 & 25 & 34 & $34 \pm 1$ & $27.1 \pm 0.4$ \\
\hline
\end{tabular}

The polymer concentration used in the different experiments equaled a) $20 \mathrm{mg} / \mathrm{mL}$, b) $1 \mathrm{mg} / \mathrm{mL}$, and c) $5 \mathrm{mg} / \mathrm{mL}$. 
Temperature at the Cloud Point $\left(T_{\mathrm{CP}}\right)$. The cloud point of the thermo-responsive polymeric surfactants was determined by monitoring the transmittance of the polymer aqueous solution as a function of temperature. Figure $6 \mathrm{~A}$ and $6 \mathrm{~B}$ show the percentage transmittance versus temperature profiles for the $\mathrm{PMEO}_{2} \mathrm{MA}_{138}$ homopolymer and the $\mathrm{PEG}_{113}-b-\mathrm{PMEO}_{2} \mathrm{MA}_{77}$ block copolymer, respectively. At low temperature, the polymer solution is clear and the transmittance takes its maximum value of $100 \%$. In the case of $\mathrm{PMEO}_{2} \mathrm{MA}_{138}$, the transmittance decreased precipitously at $26^{\circ} \mathrm{C}$ reflecting an increased turbidity of the solution. This drop in transmittance coincides with the reported LCST of $26{ }^{\circ} \mathrm{C}$ for $\mathrm{PMEO}_{2} \mathrm{MA}^{16}{ }^{16} \mathrm{In}$ Figure $6 \mathrm{~B}$, the drop in transmittance at $35{ }^{\circ} \mathrm{C}$ was attributed to the cloud point of the copolymer $\mathrm{PEG}_{113}-b-\mathrm{PMEO}_{2} \mathrm{MA}$ 77. Compared to the cloud point of $26^{\circ} \mathrm{C}$ obtained for the $\mathrm{PMEO}_{2} \mathrm{MA}_{138}$ homopolymer, the cloud point of $35^{\circ} \mathrm{C}$ found for the $\mathrm{PEG}_{113}-b-\mathrm{PMEO}_{2} \mathrm{MA}_{77}$ copolymer is higher. This difference can be explained by the presence of the hydrophilic PEG block in the copolymer which increases the solubility of the block copolymer in water, and thus its cloud point. An indication that the $\mathrm{PEG}_{113}-b-\mathrm{PMEO}_{2} \mathrm{MA}_{77}$ copolymers formed micelles came from the comparison of the trends shown in Figure 6A and 6B. It can be seen that, when the temperature passed through the cloud point, the transmittance of the $\mathrm{PMEO}_{2} \mathrm{MA}_{138}$ homopolymer solution reached $0 \%$ while that of the $\mathrm{PEG}_{113}-b-\mathrm{PMEO}_{2} \mathrm{MA}_{77}$ copolymer decreased to about $95 \%$ only. This difference in behavior is due to the more hydrophilic PEG block in the copolymer. At temperatures higher than the cloud point, the $\mathrm{PMEO}_{2} \mathrm{MA}_{138}$ homopolymer became water-insoluble and precipitated out, resulting in a milky solution. By contrast, the $\mathrm{PEG}_{113}-b-\mathrm{PMEO}_{2} \mathrm{MA} 77$ copolymer became a polymeric surfactant at temperatures above $35^{\circ} \mathrm{C}$ that formed stable micelles resulting in a translucent solution. As a 
result, the transmittance of the $\mathrm{PEG}_{113}-b-\mathrm{PMEO}_{2} \mathrm{MA}_{77}$ copolymer solution did not reach $0 \%$ but stabilized at about $95 \%$ above the cloud point.

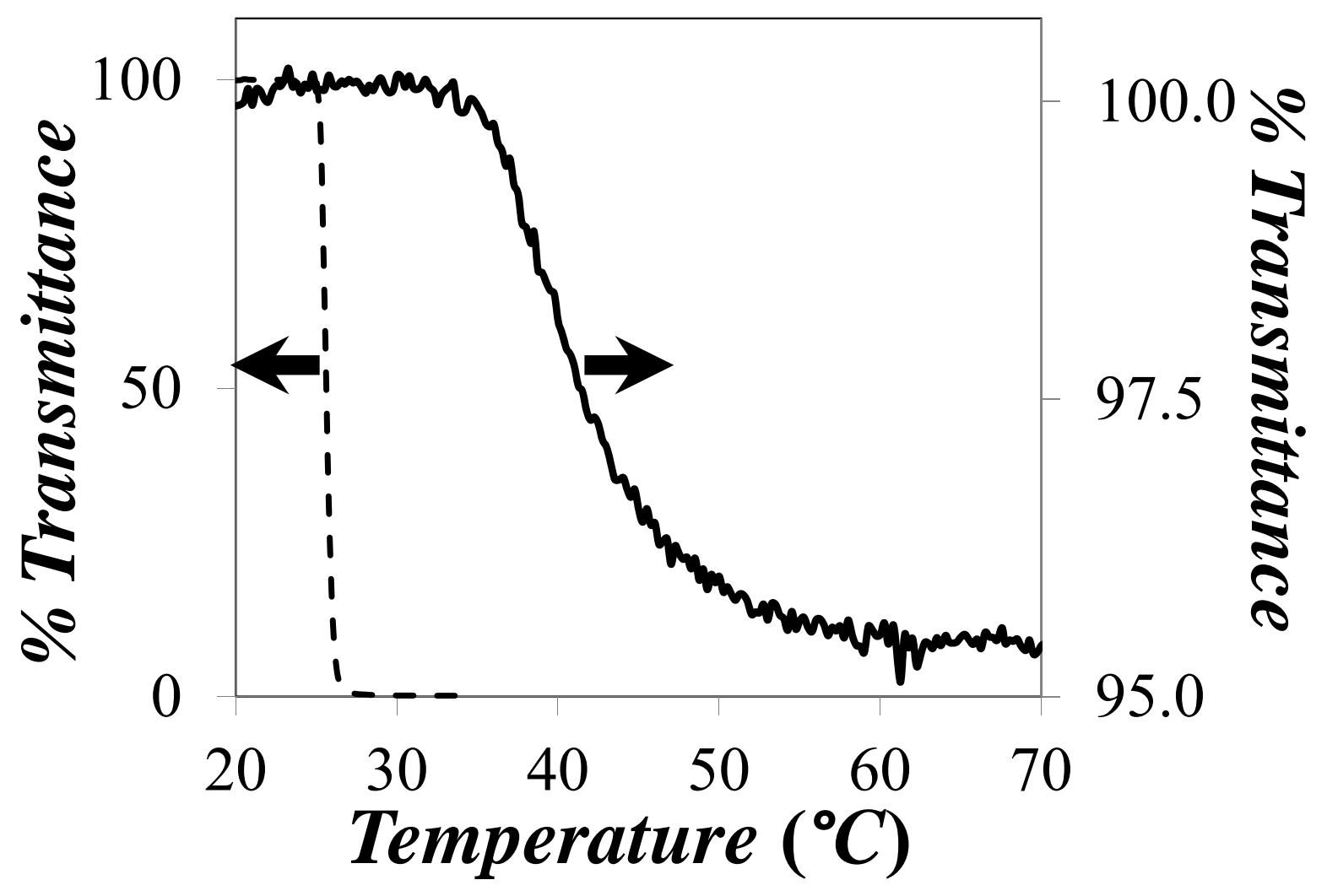

Figure 6. Plot of transmittance at $400 \mathrm{~nm}$ versus temperature for the $\mathrm{PMEO}_{2} \mathrm{MA}_{138}$ homopolymer solution (- - - ) and the $\mathrm{PEG}_{113}-b-\mathrm{PMEO}_{2} \mathrm{MA}_{77}$ copolymer solution (-).

The cloud points of the $\mathrm{PEG}_{13}-b$ - $\mathrm{PMEO}_{2} \mathrm{MAx}$ samples determined by turbidimetry are summarized in Table 1. Whereas the $\mathrm{PMEO}_{2} \mathrm{MA}_{138}$ homopolymer exhibits a cloud point of $26^{\circ} \mathrm{C}$, all copolymers had a cloud point of $34 \pm 1{ }^{\circ} \mathrm{C}$. Although the copolymers had slightly different chemical compositions with a PEG weight fraction ranging between 25 and 29\%, the small difference observed between the cloud points listed in Table 2 cannot be easily related back to the chemical composition of the copolymer. 
Dynamic Light Scattering (DLS) Measurements. Since the block copolymers underwent an LCST transition at $34 \pm 1{ }^{\circ} \mathrm{C}$ in water, dynamic light scattering (DLS) measurements were carried out to determine the size of the polymer species present in solution as a function of temperature. As shown in Figure S1, the number distribution of the hydrodynamic diameters $\left(d_{\mathrm{h}}\right)$ of the species found in the $\mathrm{PEG}_{113}-b-\mathrm{PMEO}_{2} \mathrm{MAx}$ (with $X=64,77$, and 80 ) aqueous solutions at $25,30,40$, and $50{ }^{\circ} \mathrm{C}$ showed a single peak indicating that a single species was present in solution at temperatures below and above the cloud point. The average diameter of the polymer species were plotted as a function of temperature in Figure 7. The $d_{\mathrm{h}}$ values of the $\mathrm{PEG}_{113}-b-\mathrm{PMEO}_{2} \mathrm{MAx}$ unimers in solution was small and remained constant with temperature at low temperature. At $33{ }^{\circ} \mathrm{C}$, the particle size started to increase with the block copolymers forming micelles, as expected from the cloud points of $34.0 \pm 1{ }^{\circ} \mathrm{C}$ determined by turbidimetry. The particle size increased rapidly above $33{ }^{\circ} \mathrm{C}$ reaching a maximum $d_{\mathrm{h}}$ value that remained constant at temperatures greater than $40^{\circ} \mathrm{C}$.

At low temperature, a single macromolecular species with a small $d_{\mathrm{h}}$ of about $5 \mathrm{~nm}$ was observed which would correspond to $\mathrm{PEG}_{113}-b-\mathrm{PMEO}_{2} \mathrm{MAx}$ unimers. Above the cloud point, a single larger species was observed with a $d_{\mathrm{h}}$ value of $26.3 \pm 0.3,27.1 \pm 0.4$, and 36.6 $\pm 0.3 \mathrm{~nm}$ for the $\mathrm{PEG}_{113}-b-\mathrm{PMEO}_{2} \mathrm{MA}_{64}, \mathrm{PEG}_{113}-b-\mathrm{PMEO}_{2} \mathrm{MA}_{80}$, and $\mathrm{PEG}_{113}-b-\mathrm{PMEO}_{2} \mathrm{MA}_{77}$ samples, respectively. These large $d_{\mathrm{h}}$ values would be expected for block copolymer micelles. Based on the traces shown in Figure 7, the cloud point of $\mathrm{PEG}_{13}-b-\mathrm{PMEO}_{2} \mathrm{MA} 77$ determined by DLS appears to occur at $30 \pm 5{ }^{\circ} \mathrm{C}$, which is smaller than the cloud point of $34{ }^{\circ} \mathrm{C}$ previously determined by turbidimetry (cf. Table 1 ). The difference can be explained by the 5 ${ }^{\circ} \mathrm{C}$ increment used for the DLS measurements conducted with this sample. As the $\mathrm{PEG}_{113}-b$ $\mathrm{PMEO}_{2} \mathrm{MA}_{77}$ copolymer was the first sample to be investigated, the $5{ }^{\circ} \mathrm{C}$ temperature 
increment turned out to be too large and it was adjusted to a $1{ }^{\circ} \mathrm{C}$ increment for the other $\mathrm{PEG}_{113}-b-\mathrm{PMEO}_{2} \mathrm{MAx}$ samples.

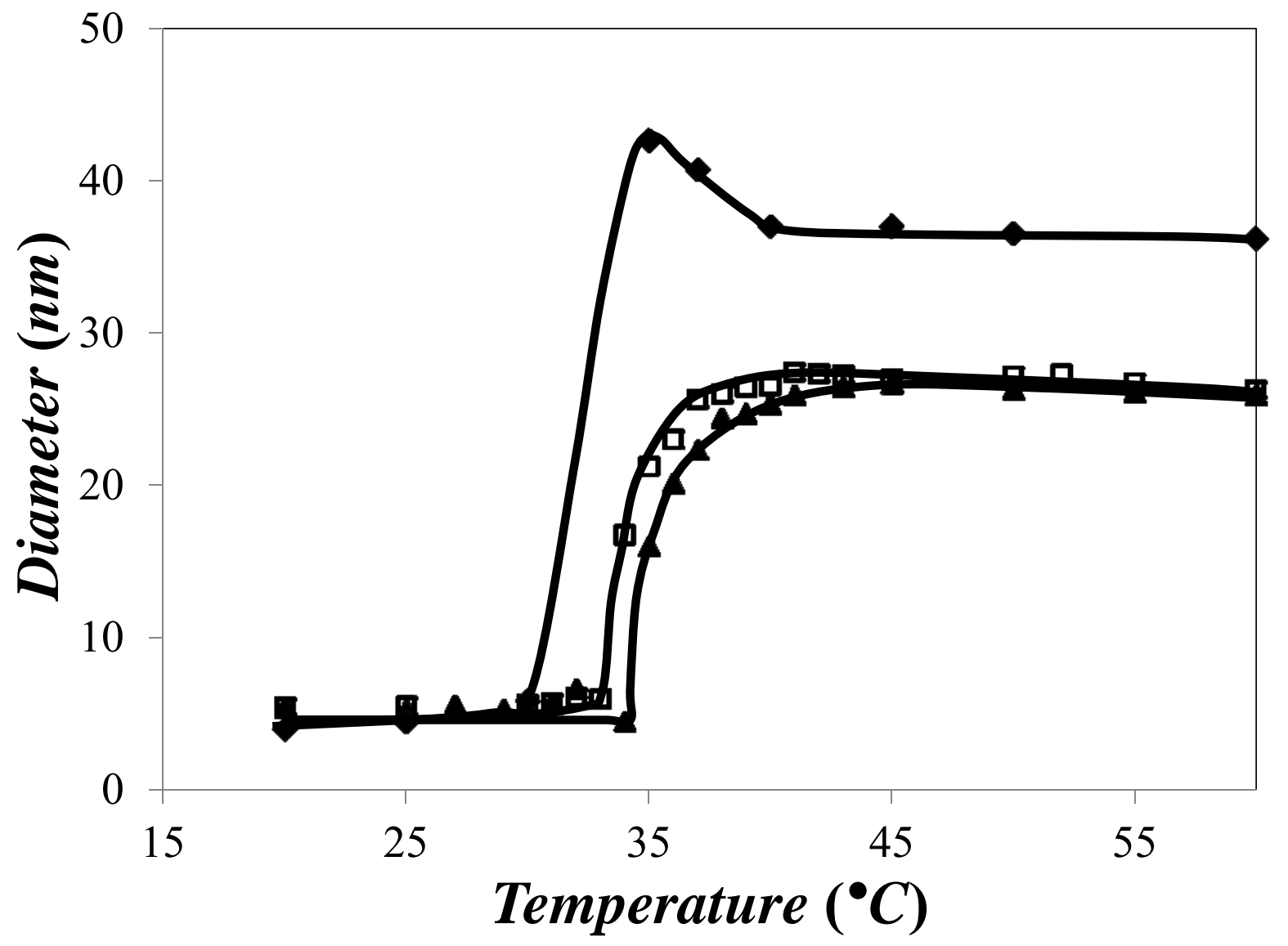

Figure 7. Plot of the hydrodynamic diameter $\left(d_{\mathrm{h}}\right)$ as a function of temperature for the block copolymers $\mathrm{PEG}_{113}-b-\mathrm{PMEO}_{2} \mathrm{MA}_{64}$ (ㅁ), $\mathrm{PEG}_{113}-b-\mathrm{PMEO}_{2} \mathrm{MA}_{77}(\diamond)$, and $\mathrm{PEG}_{113}-b-$ $\mathrm{PMEO}_{2} \mathrm{MA}_{80}(\boldsymbol{\Lambda})$ in water. Polymer concentrations equal $5 \mathrm{mg} / \mathrm{mL}$. Lines are used to guide the eye.

The $d_{\mathrm{h}}$ values of the micelles formed by the $\mathrm{PEG}_{113}-b-\mathrm{PMEO}_{2} \mathrm{MAx}$ samples listed in Table 1 suggest that the PDI of the block copolymers seems to have a strong effect on the micellar diameter, the block copolymer having the largest PDI yielding the largest block 
copolymer micelles. The increase in the $d_{h}$ value observed for the block copolymer micelles with large PDIs can be explained as follows. For larger PDIs, the shorter polymer chains locate themselves at the core-corona interface. As a result, the longer chains are squeezed out of the interfacial region and the hydrophobic blocks need to extend deeper into the core of the micelle. The process induces an enlargement of the radius of the core which is accompanied by an increase of the overall micellar dimension. This explanation is based on a study where the spacing between lamellae formed by block copolymers was found to increase as a function of the PDI of the block copolymer. ${ }^{23-25}$ The shorter chains of the distribution were found to locate themselves at the interfacial region forcing the larger chains to stretch in a process resulting in larger interlamellar distances.

Viscosity measurements. These were carried out to determine the intrinsic viscosity $[\eta]$ at $50{ }^{\circ} \mathrm{C}$ of the $\mathrm{PEG}_{113}-b-\mathrm{PMEO}_{2} \mathrm{MA}_{64}$ copolymer. Figure S2 shows plots of $\ln \left(\eta_{\mathrm{re}} / \mathrm{c}\right)$ and $\eta_{\mathrm{sp}} / \mathrm{C}$ versus the copolymer concentration (c) in $\mathrm{g} / \mathrm{mL}$. The parameters $\eta_{r e l}$ and $\eta_{s p}$ represent the relative and specific viscosity, respectively. The data shown in Figure S2 could be fitted with two straight lines that intercepted the y-axis at the same position. Their y-intercept yielded the intrinsic viscosity of the copolymer found to equal $8.1 \pm 0.2 \mathrm{~mL} / \mathrm{g}$.

After having determined the intrinsic viscosity $[\eta]$ of the $\mathrm{PEG}_{113}-b-\mathrm{PMEO}_{2} \mathrm{MA}_{64}$ solution at $50{ }^{\circ} \mathrm{C}$, the aggregation number ( $\mathrm{Nagg}$ ) of the block copolymer micelles could be estimated by using Equation 3 where the factor 2.5 comes from Einstein viscosity relation. ${ }^{26}$

$$
[\eta]=2.5 \times N_{A} \frac{V_{h}}{M_{n, \text { mic }}}=2.5 \times N_{A} \frac{V_{h}}{N_{a g g} M_{n}}
$$

In Equation 3, $N_{A}$ is Avogadro's number, $V_{h}, M_{n, m i c}$, and $N_{a g g}$ are, respectively, the hydrodynamic volume, the molecular weight, and the aggregation number of a block 
copolymer micelle, and $M_{n}$ is the number-average molecular weight of the copolymer. Since $V_{h}$ can be calculated from the hydrodynamic diameter $\left(d_{h}\right)$ of the block copolymer micelles found to equal $26.3 \mathrm{~nm}$ from DLS measurements and [ $\eta$ ] was found to equal $8.1 \mathrm{~mL} / \mathrm{g}, N_{\text {agg }}$ in Equation 3 was determined to equal $100 \pm 8$ for $\mathrm{PEG}_{113}-b-\mathrm{PMEO}_{2} \mathrm{MA}_{64}$.

Oil Extraction. The cloud point of the $\mathrm{PEG}_{113}-b-\mathrm{PMEO}_{2} \mathrm{MAx}$ solutions determined by turbidimetry equaled $34 \pm 1{ }^{\circ} \mathrm{C}$, which corresponds to the temperature at which micelles start to form, while the plot obtained by DLS for the block copolymers in Figure 7 indicates that micelle formation is complete at temperatures greater than $45^{\circ} \mathrm{C}$. As the temperature of the aqueous solution is increased past $34 \pm 1{ }^{\circ} \mathrm{C}$, micelle formation is induced by the dehydration of the $\mathrm{PMEO}_{2} \mathrm{MA}$ blocks and their subsequent aggregation into hydrophobic aggregates that are stabilized by the $\mathrm{PEG}_{113}$ blocks. As more micelles generate more hydrophobic domains in the solution, a temperature of either 45 or $50^{\circ} \mathrm{C}$ was selected for oil extraction to ensure the formation of a large number of block copolymer micelles.

Extraction Efficiencies. A set of extractions were first conducted with aqueous solutions of the different block copolymers and their constituting homopolymers without any toluene added to the mixtures. The results from these extractions are summarized in Figure 8. Figure 8A shows the results obtained for the aqueous solutions used for oil extraction experiments without toluene. Their composition is listed hereafter starting from the left side of the figure: pure water, $100 \mathrm{mM}$ sodium dodecyl sulfate (SDS) aqueous solution, and $1 \mathrm{mg} / \mathrm{mL}$ aqueous solutions of PEG homopolymer, $\mathrm{PMEO}_{2} \mathrm{MA}$ homopolymer, $\mathrm{PEG}_{113}-b-\mathrm{PMEO}_{2} \mathrm{MA}_{77}$ block copolymer, and poly( $N$-isopropyl acrylamide) (PNIPAM) homopolymer. SDS was selected for comparison as it is a typical surfactant. PNIPAM was introduced in this comparison since it is a well-known thermo-responsive polymer with an LCST of $32{ }^{\circ} \mathrm{C}$ in water. Visual 
inspection of the vials led to the obvious conclusion that without toluene, none of these aqueous solutions could extract the oil from the oil sands efficiently. This conclusion was reached by noting the extremely thin oil layer at the top of the aqueous solution and the big oil blobs remaining at the bottom of the vials. In other words, all the aqueous solutions investigated in Figure 8A extracted very little oil from the oil sands if an aqueous solution of the polymers alone was used in the extraction. To improve the efficiency of oil extraction, a small amount of toluene was added to the aqueous solution as described in the Experimental Section for the Extraction Protocol. Toluene was selected because Athabasca oil is known to have a large aromatic content of $40 \mathrm{wt} \%{ }^{27}$ The addition of $60 \mathrm{mg}$ of toluene resulted in a significant improvement in oil extraction efficiency, as illustrated in Figure 8B. The aromatic character of toluene appeared to enhance oil extraction from the oil sands. ${ }^{8,27}$

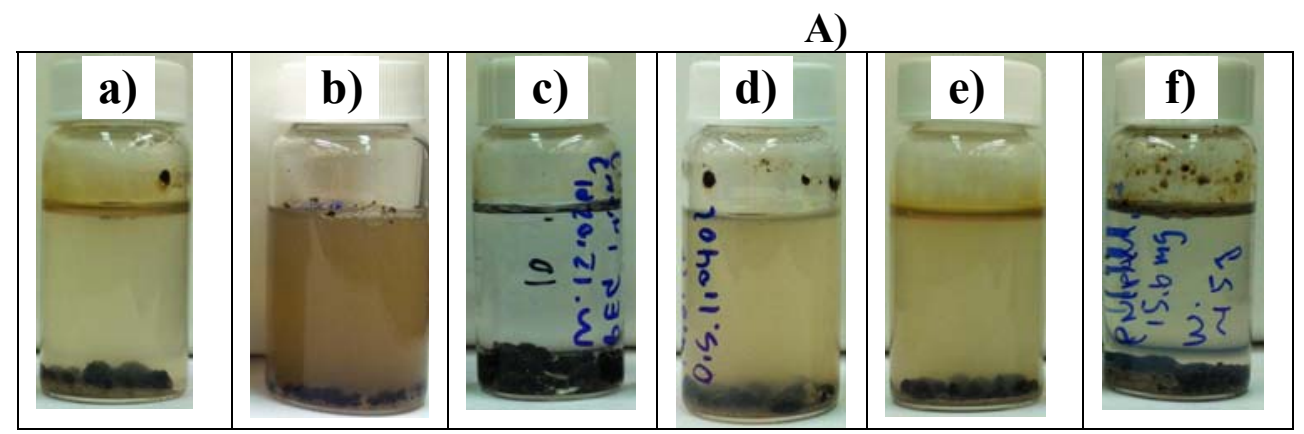

B)

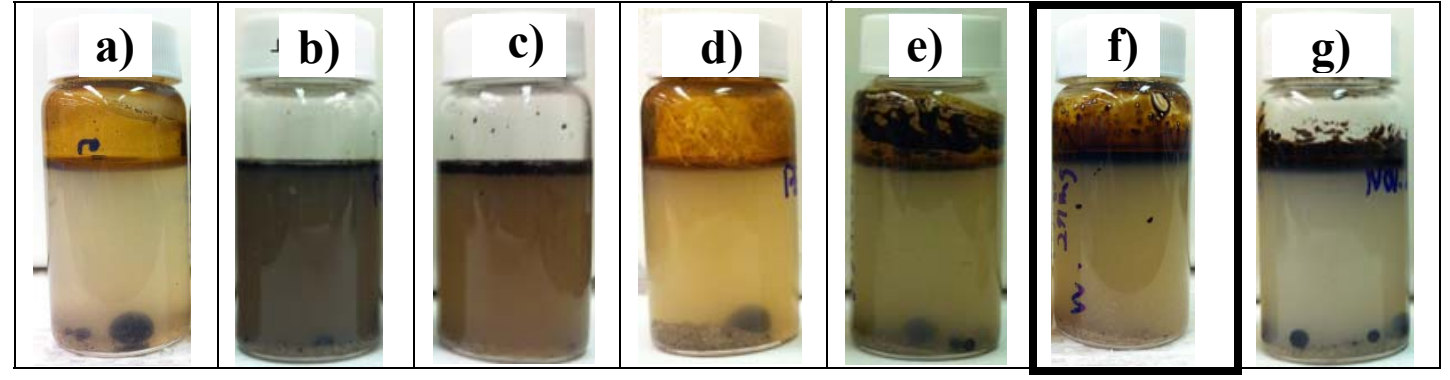

Figure 8. A) Extraction without toluene: a) pure water, b) $100 \mathrm{mM}$ SDS, and $1 \mathrm{mg} / \mathrm{mL}$ aqueous solutions of c) PEG, d) PMEO 2 MA, e) PEG $113-b-\mathrm{PMEO}_{2} \mathrm{MA} 77$, and f) PNIPAM. B) Extraction with $60 \mathrm{mg}$ toluene: a) pure water, b) $100 \mathrm{mM}$ SDS, c) $200 \mathrm{mM}$ SDS, and 1 
$\mathrm{mg} / \mathrm{mL}$ aqueous solutions of d) $\mathrm{PEG}$, e) $\mathrm{PMEO}_{2} \mathrm{MA}$, f) $\mathrm{PEG}_{113}-b-\mathrm{PMEO}_{2} \mathrm{MA}_{77}$, and g) PNIPAM. Mixtures were shaken for $24 \mathrm{hrs}$ at $\mathrm{T}=45^{\circ} \mathrm{C}$.

As shown in Figure 8B, the oily layer at the top of the aqueous solution was thicker and fewer black oil blobs were found at the bottom of the vials. Most importantly, it was noticeable that the aqueous solution of $\mathrm{PEG}_{113}-b-\mathrm{PMEO}_{2} \mathrm{MA}_{77}$ copolymer resulted in the most efficient extraction: A very thick layer of oil could be found at the top of the aqueous layer, while no black oil blobs remained at the bottom of the vial. As a matter of fact, a $100 \%$ extraction yield was obtained in this case. Comparison of the extraction results obtained in Figure $8 \mathrm{~A}$ and $8 \mathrm{~B}$ led to the conclusion that using $15 \mathrm{~mL}$ of a $1 \mathrm{mg} / \mathrm{mL} \mathrm{PEG}_{113}-b-$ $\mathrm{PMEO}_{2} \mathrm{MA}_{77}$ aqueous solution with $60 \mathrm{mg}$ of toluene yielded the most efficient extraction.

Compared to the block copolymer which resulted in a well-defined phase separation between the oil and aqueous solution, the aqueous solutions with the molecular surfactant SDS seemed to stabilize the oil in the aqueous solution instead of leading to oil extraction, an undesired outcome. Also the other thermoresponsive polymer PNIPAM tried in this study did not appear to extract the oil from the oil sands efficiently on its own.

Optimization of the Amount of Toluene Used in the Extraction Process. As mentioned earlier, the results shown in Figure 8B demonstrated the superiority of the $1 \mathrm{mg} / \mathrm{mL}$ PEG113-b$\mathrm{PMEO}_{2} \mathrm{MA} 77$ aqueous solution at extracting oil by adding a small amount (60 mg) of toluene. This conclusion was further confirmed by monitoring the extraction efficiency $\left(E_{\mathrm{ex}}\right)$ as a function of the mass of toluene $\left(m_{\mathrm{tol}}\right)$ added at the top of $15 \mathrm{~mL}$ of either pure water or a 1 $\mathrm{mg} / \mathrm{mL} \mathrm{PEG}_{113}-b-\mathrm{PMEO}_{2} \mathrm{MA} 77$ aqueous solution. $E_{\text {ex }}$ was determined by taking the ratio of the amount of oil recovered per gram of oil sand divided by the known oil content of the oil 
sands equal to $110 \mathrm{mg} / \mathrm{g}$ as determined by Soxhlet extraction. A plot of $E_{\mathrm{ex}}$ versus $m_{\mathrm{tol}}$ is shown in Figure 9A.

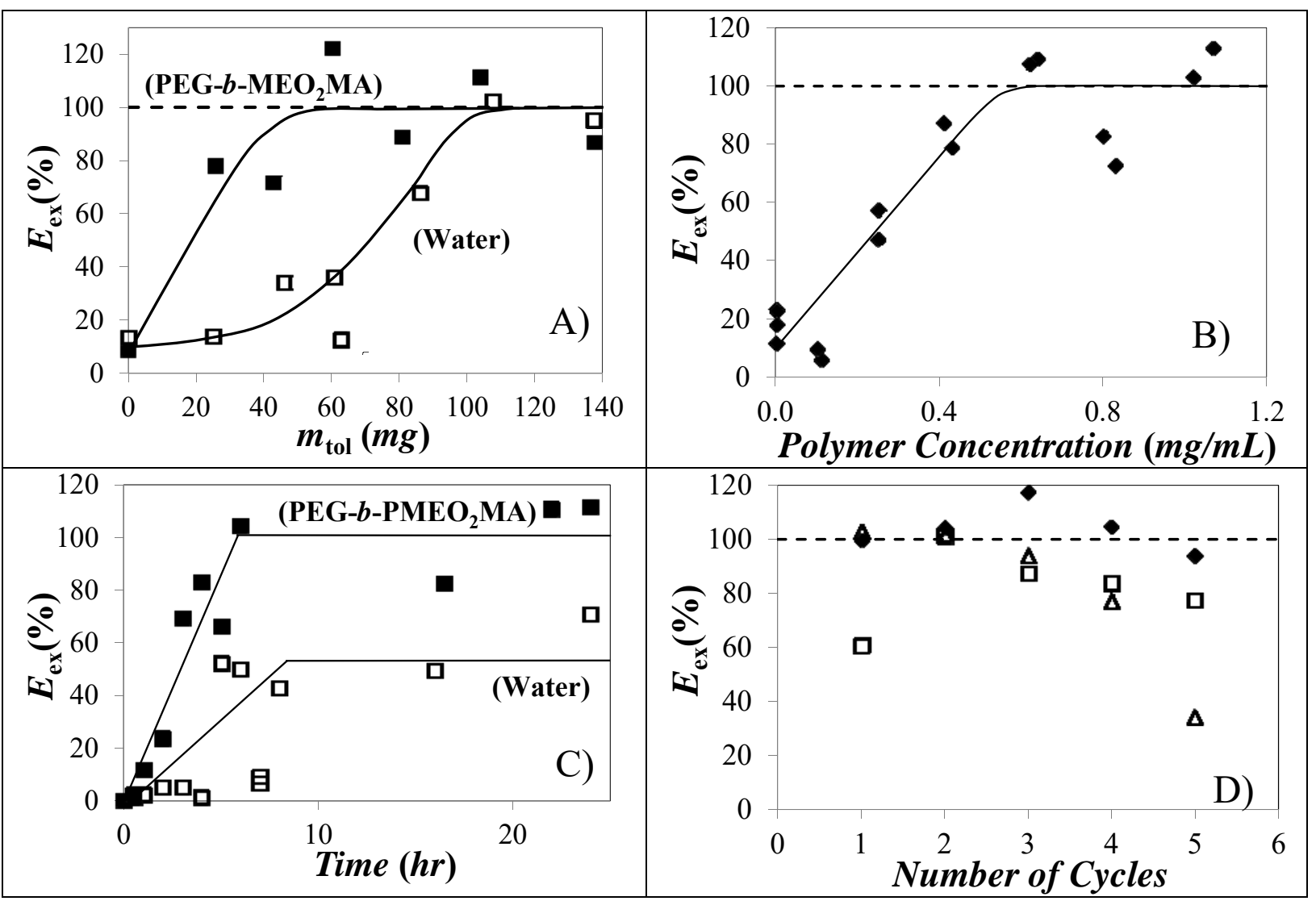

Figure 9. A) Plot of the extraction efficiency $\left(E_{e x}\right)$ versus the mass of toluene $\left(m_{\text {tol }}\right)$ added to

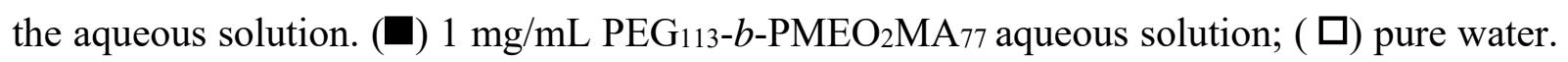
B) Plot of $E_{\text {ex }}$ as a function of $\mathrm{PEG}_{113}-b-\mathrm{PMEO}_{2} \mathrm{MA}_{80}$ concentration when the extraction is conducted with $65 \mathrm{mg}$ of toluene. C) Plot of $E_{e x}$ versus extraction time when the extraction is

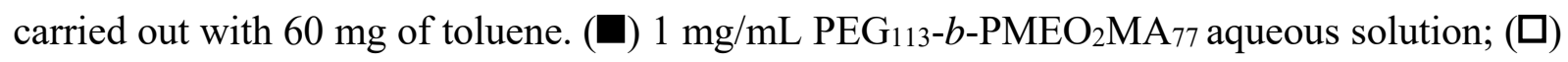
pure water. D) Plot of $E_{e x}$ versus the number of extraction cycles using PEG $_{113-b}-$ $\mathrm{PMEO}_{2} \mathrm{MA}_{66}$ and $65 \mathrm{mg}$ of toluene. ( $\square$ ) Sample \#1, $(\Delta)$ Sample \#2, and $(\nabla)$ Sample \#3. $T_{\mathrm{ex}}=50^{\circ} \mathrm{C}$. 
For $m_{\text {tol }}$ greater than or equal to $100 \mathrm{mg}, E_{\mathrm{ex}}$ reached unity within experimental error indicating complete oil recovery. It must be pointed out that some of the extraction experiments yielded $E_{\text {ex }}$ values greater than unity. These results were attributed to the collection of some sand particles with the oil that artificially increased the weight of the recovered oil. However, for all other $m_{\text {tol }}$ smaller than $100 \mathrm{mg}, E_{\text {ex }}$ determined with the 1 $\mathrm{mg} / \mathrm{mL} \mathrm{PEG}_{113}-b-\mathrm{PMEO}_{2} \mathrm{MA}_{77}$ aqueous solution was consistently larger than $E_{\text {ex }}$ obtained with water alone. The trend shown in Figure 9A demonstrates that the presence of $1 \mathrm{mg} / \mathrm{mL}$ $\mathrm{PEG}_{113}-b-\mathrm{PMEO}_{2} \mathrm{MA}_{77}$ in the aqueous solution enhances oil extraction substantially compared to extractions carried out in pure water. It also suggests that when using more than $60 \mathrm{mg}$ of toluene with $1 \mathrm{mg} / \mathrm{mL} \mathrm{PEG}_{113}-b-\mathrm{PMEO}_{2} \mathrm{MA}_{77}$ aqueous solution, $100 \%$ oil recovery can be expected. Based on the results obtained in Figure 9A, all extractions conducted in the remainder of the study used 60 or $65 \mathrm{mg}$ of toluene.

Effect of Polymer Concentration on Extraction Yield. Extractions were carried out with aqueous solutions prepared over a range of block copolymer concentrations. The extraction efficiency was low at low block copolymer concentration, but the recovery yield increased with increasing block copolymer concentration in Figure 9B, reaching optimum recoveries for block copolymer concentrations of $0.4 \mathrm{mg} / \mathrm{mL}$ or greater. In most extraction experiments, a block copolymer concentration of $1 \mathrm{mg} / \mathrm{mL}$ was used to ensure maximum extraction yield. Effect of Extraction Time on Extraction yield. Time-dependent experiments were carried out to determine the minimum time required for a complete oil extraction cycle. A plot of $E_{e x}$ versus time from 0 to $24 \mathrm{hrs}$ is shown in Figure 9C. In Figure 9C, the Eex value obtained with the $1 \mathrm{mg} / \mathrm{mL} \mathrm{PEG}_{113}-b-\mathrm{PMEO}_{2} \mathrm{MA}_{77}$ aqueous solution increased continuously with time during the first 6 hrs, reaching complete oil recovery after 6 hrs and remaining constant 
afterwards. By comparison, $E_{\text {ex }}$ obtained for pure water was lower at all extraction durations, and maximum recovery was also achieved after about $6 \mathrm{hrs}$. In conclusion, the trend shown in Figure $9 \mathrm{C}$ indicates that the $1 \mathrm{mg} / \mathrm{mL} \mathrm{PEG}_{113}-b-\mathrm{PMEO}_{2} \mathrm{MA}_{77}$ aqueous solution resulted in a more efficient oil recovery as compared to pure water, and that maximum recovery was achieved after 6 hrs.

Oil Extraction Efficiency as a Function of the Number of Extraction Cycles. The extraction scheme shown in Figure 1 suggests that the main advantage of using an aqueous solution of thermo-responsive polymeric surfactants consists in the ability to reuse their solution to achieve consecutive extractions. In reality, a certain amount of the $\mathrm{PEG}_{113}-b-\mathrm{PMEO}_{2} \mathrm{MAx}$ block copolymers is bound to interact with the organic phase (toluene, oil, or the sand particles), leading to a decrease in polymer concentration which might have a detrimental effect on the efficiency of oil extraction. A plot of $E_{e x}$ versus the number of extraction cycles is shown in Figure 9D. These experiments were conducted in triplicate to gauge the reproducibility of the extraction protocol. Out of 15 data points resulting from the 5 extraction cycles carried out in triplicate, 13 yielded an $E_{\text {ex }}$ value between 77 and $117 \%$ reflecting efficient oil recovery. Two data points, one with an $E_{e x}$ of $60 \%$ after the first extraction cycle and another with an $E_{\text {ex }}$ of $34 \%$ after the fifth extraction cycle seemed to be outliers. A slight decrease in Eex was observed for the fourth and fifth cycles. This might be due to the gradual loss of $\mathrm{PEG}_{113-}-\mathrm{b}-\mathrm{PMEO}_{2} \mathrm{MA}_{64}$ copolymer that must occur with increasing number of oil extraction cycles. The loss of $\mathrm{PEG}_{113}-b-\mathrm{PMEO}_{2} \mathrm{MA}_{64}$ copolymer after an extraction cycle will be confirmed in the following section. At this stage, it can be concluded that starting with a 1 $\mathrm{mg} / \mathrm{mL} \mathrm{PEG}_{113}-b-\mathrm{PMEO}_{2} \mathrm{MA}_{64}$ aqueous solution enabled a high oil recovery even after 5 consecutive extraction cycles. 
Recovery of block copolymer after Oil Extraction. Three vials containing $65 \mathrm{mg}$ of toluene, $15 \mathrm{~g}$ of a $1 \mathrm{mg} / \mathrm{mL} \mathrm{PEG}_{113}-\mathrm{b}-\mathrm{PMEO}_{2} \mathrm{MA}_{80}$ aqueous solution, and $1 \mathrm{~g}$ of oil sands were prepared and placed in the shaker for $24 \mathrm{hrs}$. After oil extraction, the aqueous layer of the three samples was collected and freeze-dried, and the lyophilized polymer was dissolved in THF. This solution was injected in the GPC instrument and its DRI intensity was determined. The DRI signals of the GPC traces obtained for the three samples are shown in Figure S4 and their MWD was compared to that of the block copolymer before extraction. $\mathrm{PEG}_{113}-b$ $\mathrm{PMEO}_{2} \mathrm{MA}_{80}$ before and after bitumen extraction all eluted at $24 \mathrm{~mL}$ yielding similar MWDs with an $\mathrm{M}_{\mathrm{n}}$ value of 21,000 $\pm 1,000 \mathrm{~g} / \mathrm{mol}$ and a PDI value of $1.2 \pm 0.0$. Together, the similar MWDs recovered for the block copolymer before and after bitumen extraction suggest that $\mathrm{PEG}_{113-}-\mathrm{P}-\mathrm{PMEO}_{2} \mathrm{MA} 80$ was not degraded after one extraction cycle. The percentage of block copolymer recovered for these three samples, calculated from the calibration curve shown in Figure S3 equaled $0.83,0.73$, and 0.79 . Therefore, the fraction of block copolymer recovered after one oil extraction cycle was determined to equal $0.78 \pm 0.05$, which indicates that each extraction cycle results in a $22 \%$ loss of $\mathrm{PEG}_{113}-b-\mathrm{PMEO}_{2} \mathrm{MA} 80$. However, it also indicates that $78 \%$ of polymer remains in the aqueous solution for the next cycle.

These experiments also substantiate the fact that a relatively good oil recovery was obtained after 5 extraction cycles starting with a $1 \mathrm{mg} / \mathrm{mL}$ block copolymer concentration. Based on the block copolymer recovery yield of $78 \%$ after one extraction, the block copolymer concentration for the fifth extraction would be expected to equal $0.78^{4} \times(1 \mathrm{mg} / \mathrm{mL})$ $=0.37 \mathrm{mg} / \mathrm{mL}$. Based on the trend shown in Figure 9B, such a block copolymer concentration is expected to result in a satisfying oil recovery even after five extraction cycles as was found in Figure 9D. 
Toluene-block copolymer Interactions: Experiments conducted thus far have confirmed the importance of toluene in the extraction process (Figures 8 and 9). This observation suggests that the block copolymers interact to some extent with toluene. To investigate this possibility, $100 \mu \mathrm{L}$ of a $5 \mathrm{mg} / \mathrm{mL}$ aqueous solution of $\mathrm{PEG}_{13}-b-\mathrm{PMEO}_{2} \mathrm{MA}_{80}$ was probed by DLS as a function of temperature as $0.5 \mu \mathrm{L}$ of toluene was placed at the surface of the solution. A single species was found in solution and its $d_{\mathrm{h}}$ value was determined and plotted as a function of temperature in Figure 10.

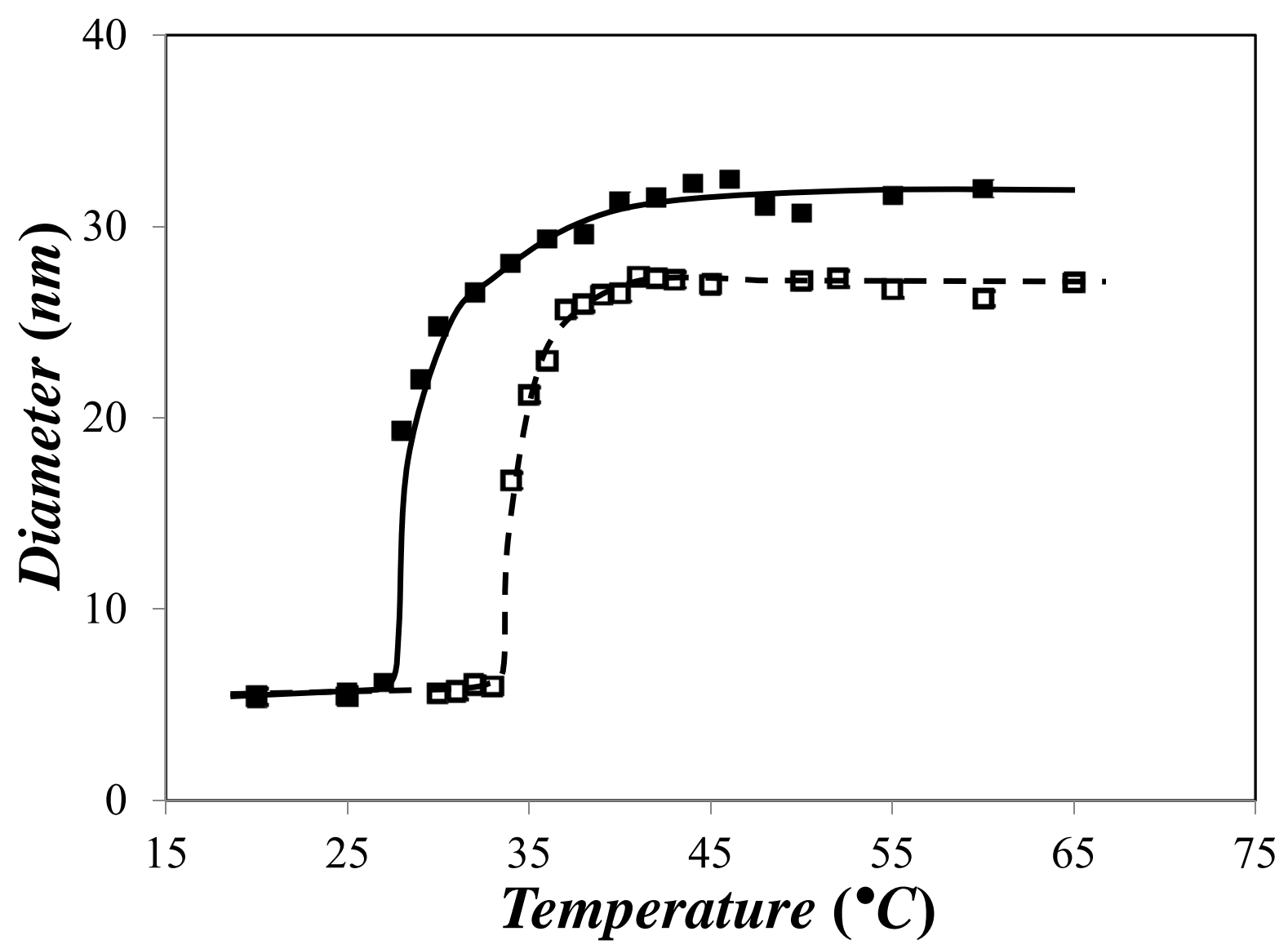

Figure 10. Plot of $D_{\mathrm{h}}$ as a function of temperature for a $5 \mathrm{mg} / \mathrm{mL} \mathrm{PEG}_{113}-b-\mathrm{PMEO}_{2} \mathrm{MA}_{80}$ aqueous solution with $(\square)$ or without $(\square)$ toluene. Lines are used to guide the eye. 
The trends shown in Figure 10 indicate that the presence of toluene in the aqueous solution lowers $T_{\mathrm{C}}$ from $33{ }^{\circ} \mathrm{C}$ to $27{ }^{\circ} \mathrm{C}$ and increases $d_{\mathrm{h}}$ for the block copolymer micelles from $27.1 \pm 0.4$ to $31.6 \pm 0.9 \mathrm{~nm}$. This $17 \%$ increase in $d_{\mathrm{h}}$ is equivalent to a $60 \%$ increase in micellar volume implying that the block copolymer micelles are swollen with toluene and confirming their role in shuttling toluene from the surface of the aqueous solution to the bottom of the vial where toluene can interact with the oil surrounding the sand particles. The affinity of the $\mathrm{PMEO}_{2} \mathrm{MA}$ block with toluene, and more generally the thermo-responsive polymeric surfactant and the organic thinner used in an extraction, might represent an important feature to consider when designing thermo-responsive polymeric surfactants for oil extraction.

To further illustrate the importance of interactions between the organic thinner and the block copolymer, an extraction was carried out with $60 \mathrm{mg}$ of dodecane instead of toluene. Whereas $1 \mathrm{mg}$ of block copolymer dissolved readily in $1 \mathrm{~mL}$ of toluene, it was insoluble in 1 $\mathrm{mL}$ of dodecane. The result of the extraction is shown in Figure 11 where the oil sand remained untouched at the bottom of the vial. Since dodecane did not interact with the block copolymer, it remained at the surface of the aqueous solution and never came in contact with the oil.

The results shown in Figures 10 and 11 strongly suggest that a successful extraction requires that the organic thinner be brought into contact with the oil sand. The block copolymers which are formed above $T_{\mathrm{c}}$ appear to shuttle the organic thinner from the surface of the aqueous solution to the bottom of the vial where it interacts with the oil and enables loading of the micelles with the oil. In turn, the micelles laced with oil diffuse back to the airwater interface where they unload their cargo at the surface of the aqueous solution. The 
process continues until all the oil has been extracted from the oil sand. Whatever oil remains trapped in the block copolymer micelles at the end of the extraction can be recovered by bringing the solution temperature below its cloud point. The micelles decompose and release the individual block copolymers into the solution which allows the oil to diffuse to the surface.

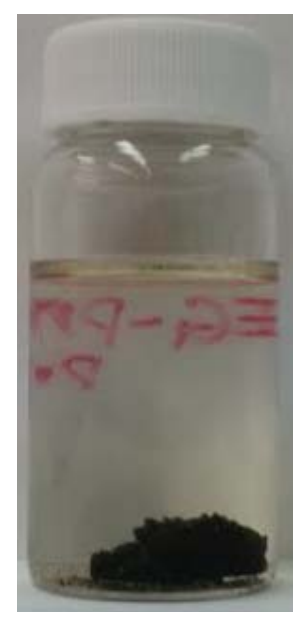

Figure 11. Result of the extraction of oil from oil sands using $60 \mathrm{mg}$ of dodecane instead of toluene. $T_{\mathrm{ex}}=50{ }^{\circ} \mathrm{C}$.

\section{CONCLUSIONS}

$\mathrm{PEG}_{113}-b$-PMEO $2 \mathrm{MAx}$ samples with $X=48,64,77$, and 80 were synthesized by atom transfer radical polymerization (ATRP) and shown to form monodisperse block copolymer micelles at temperatures greater than the cloud point of the $\mathrm{PMEO}_{2} \mathrm{MA}$ block. At temperatures above $50{ }^{\circ} \mathrm{C}$, the block copolymer micelles assisted the back-and-forth transfer of toluene from the surface of the aqueous solution to the bottom of the vial where toluene could solvate the oil and shuttle it through the aqueous phase back to the surface where the oil would accumulate. Cooling the solution to room temperature that is lower than the cloud point of the block copolymer solution restored the solubility of the block copolymer in water, destabilized the 
emulsion that was generated at $50{ }^{\circ} \mathrm{C}$ with the oil-saturated toluene droplets stabilized by the block copolymers, and induced the toluene laced with oil to phase separate and accumulate at the surface leaving behind the pristine sand at the bottom of the vial (see Figure 2). The oil that gathered at the surface of the vial was collected and the block copolymer solution could be used in another cycle of extraction.

Although the chemical composition of the $\mathrm{PEG}_{113}-b-\mathrm{PMEO}_{2} \mathrm{MAx}_{\mathrm{x}}$ block copolymers might not be robust enough to ensure their applicability in an industrial setting, this study demonstrates several advantages associated with the use of thermo-responsive polymeric surfactants in the extraction of oil from oil sands. First, the aqueous block copolymer solutions can be re-used for repeated extraction cycles thus minimizing water waste. Second, the ability to destabilize the oil-in-water emulsion that is generated during the extraction process by simply lowering the temperature of the solution should minimize the generation of tailing ponds, a persistent environmental hazard. Third, the procedure introduced in this study is based on the use of heat, an organic thinner (toluene), and water which are all typical elements found in any current extraction process making it easier to apply in an industrial setting. In short, the use of thermo-responsive polymeric surfactants opens new research venues for the extraction of oil from oil sands.

\section{ACKNOWLEDGEMENTS}

BY and JD are thankful to funding from Imperial Oil and NSERC and the help of Thomas Gibson, Junhui Xu, and Lu Li. 


\section{SUPPORTING INFORMATION}

Histograms of DLS of block copolymer solutions as a function of temperature, plot to determine intrinsic viscosity, and calibration curve relating DRI signal to polymer concentration. This information is free of charge via the Internet at http://pubs.acs.org/.

\section{REFERENCES}

1. Camp, F. W. Tar Sands of Alberta, Canada, Third Edition; Cameron Engineers Inc.; Denver, CO, 1976.

2. Masliyah, J.; Zhou, Z.; Xu, Z.; Czarnecki, J.; Hamza, H. Understanding of Water-Based Bitumen Extraction from Athabasca Oil Sands. Can. J. Chem. Eng. 2004, 82, 628-654.

3. Saniere, A.; Hénaut, I.; Argillier, J. F. Pipeline Transportation of Heavy Oils, a Strategic, Economic and Technological Challenge. Oil Gas Sci. Technol. 2004, 59, 455466.

4. Larter, S.; Adams, J.; Gates, I. D.; Bennett, B.; Huang, H. The Origin, Prediction and Impact of Oil Viscosity Heterogeneity on the Production Characteristics of Tar Sand and Heavy Oil Reservoirs. Annu. Tech. Meet. Pet. Soc. CIM, [Prepr.] 2006, 134.

5. Reichert, C.; Fuhr, B.; Sawatzky, H.; Lafleur, R.; Verkoczy, B.; Soveran, D.; Jha, K. Fire Flood Recovery Process Effects upon Heavy Oil Properties. Fuel Sci. Technol. Int. 1989, 7, 851-878.

6. Craig, F. F.; Parrish, D. R. A Multipilot Evaluation of the COFCAW Process. J. Pet. Technol. 1974, 26, 659-666. 
7. Carrigy, M. A. Ed. Athabasca Oil Sands: A Collection of Papers Presented to K. A. Clark on the 75th Anniversary of his Birthday. Research Council of Alberta, Edmonton, Alberta, Canada, 1963, 123-141.

8. Sury, K. N. Low Temperature Bitumen Recovery Process. US Patent 4946 597, 1990.

9. Sury, K. N. Low Temperature Bitumen Recovery Process. Canadian Patent 1302 327, 1992.

10. Yang, H.; Wang, Y.; Ding, M.; Hu, B.; Ren, S. Water-Assisted Solvent Extraction of Bitumen from Oil Sands. Ind. Eng. Chem. Res. 2012, 51, 3032-3038.

11. Rao, F; Liu, Q. Froth Treatment in Athabasca Oil Sands Bitumen Recovery Process: a Review. Energy Fuels 2013, 27, 7199-7207.

12. Kirpalani, D. M.; Matsuoka, A. CFD Approach for Simulation of Bitumen Froth Settling Process - Part I: Hindered Settling of Aggregates. Fuel 2008, 87, 380-387.

13. Long, Y.; Dabros, T.; Hamza, H. Stability and Settling Characteristics of SolventDiluted Bitumen Emulsions. Fuel 2002, 81, 1945-1952.

14. Park, M. H.; Joo, M. K.; Choi, B. G.; Jeong, B. Biodegradable Thermogels. Acc. Chem. Res. 2011, 45, 424-433.

15. Bonacucina, G.; Cespi, M.; Mencarelli, G.; Giorgioni, G.; Palmieri, G. F. Thermosensitive Self-Assembling Block Copolymers as Drug Delivery Systems. Polymers 2011, 3, 779-811.

16. Lutz, J.-F.; Hoth, A. Preparation of Ideal PEG Analogues with a Tunable Thermosensitivity by Controlled Radical Copolymerization of 2-(2Methoxyethoxy)ethyl Methacrylate and Oligo(ethylene glycol) Methacrylate. Macromolecules 2006, 39, 893-896. 
17. Lutz, J.-F.; Akdemir, O.; Hoth, A. Point by Point Comparison of two Thermosensitive Polymers Exhibiting a Similar LCST: Is the Age of Poly(NIPAM) Over? J. Am. Chem. Soc. 2006, 128, 13046-13047.

18. Mei, A.; Guo, X.; Ding, Y; Zhang, X; Xu, J; Fan, Z; Du, B. PNIPAm-PEO-PPO-PEOPNIPAm Pentablock Terpolymer: Synthesis and Chain Behavior in Aqueous Solution. Macromolecules 2010, 43, 7312-7320.

19. Kitano, H.; Kondo, T.; Suzuki, H.; Ohno, K. J. Temperature-Responsive Polymer-Brush Constructed on a Glass Substrate by Atom Transfer Radical Polymerization. Colloid Interface Sci. 2010, 345, 325-331.

20. Kitano, H.; Hirabayashi, T.; Gemmei-Ide, M; Kyogoku, M. Effect of Macrocycles on the Temperature-Responsiveness of Poly[(methoxy diethylene glycol methacrylate)graft-PEG]. Macromol. Chem. Phys. 2004, 205, 1651-1659.

21. Jacobs, F. S.; Filby, R. H. Solvent Extraction of Oil-Sand Components for Determination of Trace Elements by Neutron Activation Analysis. Anal. Chem. 1983, $55,74-77$.

22. Dust, J. M.; Fang, Z.-H.; Harris, J. M. Proton NMR Characterization of Poly(ethylene glycols) and Derivatives. Macromolecules 1990, 23, 3742-3746.

23. Noro, A.; Cho, D.; Takano, A.; Matsushita, Y. Effect of Molecular Weight Distribution on Microphase-Separated Structures from Block Copolymers. Macromolecules 2005, $38,4371-4376$.

24. Mayes, A. M.; Russell, T. P.; Satija, S. K.; Majkrzak, C. F. Homopolymer Distributions in Ordered Block Copolymers. Macromolecules 1992, 25, 6523-6531. 
25. Torikai, N.; Takabayashi, N.; Noda, I.; Koizumi, S.; Mori, Y.; Matsushita, Y. Lamellar Domain Spacings of Diblock Copolymer/Homopolymer Blends and Conformations of Block Chains in their Microdomains. Macromolecules 1997, 30, 5698-5703.

26. Flory, P. J. Principles of Polymer Chemistry Cornell University Press, Ithaca, 1953, pp 605-606.

27. Rao, F.; Liu, Q. Froth Treatment in Athabasca Oil Sands Bitumen Recovery Process: A Review. Energy Fuels 2013, 27, 7199-7207. 
Table of Content

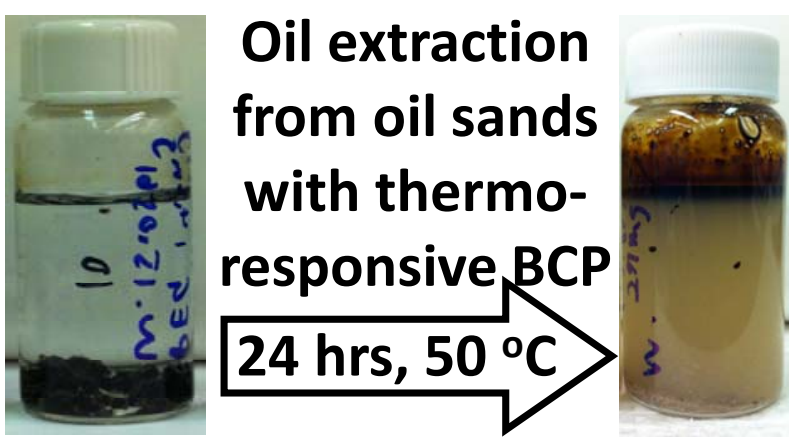

